\title{
Nuclear reprogramming: the zygotic transcription program is established through an "erase-and-rebuild" strategy
}

Feng Sun ${ }^{1, *}$, Haiyan Fang ${ }^{2, *}$, Ruizhen $\mathrm{Li}^{2, *}$, Tianlong Gao ${ }^{1}$, Junke Zheng ${ }^{2}$, Xuejin $\mathrm{Chen}^{2}$, Wenqin Ying ${ }^{2}$, Hui Z Sheng $^{2}$

${ }^{1}$ Program for Graduation Studies, Shanghai Institutes for Biological Sciences, Chinese Academy of Sciences, Shanghai 200031, China; ${ }^{2}$ Center for Developmental Biology, Xinhua Hospital, School of Medicine, Shanghai Jiao Tong University, 1665 Kong Jiang Road, Shanghai 200092, China

Oocytes display a maternal-specific gene expression profile, which is switched to a zygotic profile when a haploid set of chromatin is passed on to the fertilized egg that develops into an embryo. The mechanism underlying this transcription reprogramming is currently unknown. Here we demonstrate that by the time when transcription is shut down in germinal vesicle oocytes, a range of general transcription factors and transcriptional regulators are dissociated from the chromatin. The global dissociation of chromatin factors $(\mathrm{CFs})$ disrupts physical contacts between the chromatin and $\mathrm{CFs}$ and leads to erasure of the maternal transcription program at the functional level. Critical transcription factors and regulators remain separated from chromatin for a prolonged period, and become re-associated with chromatin shortly after pronuclear formation. This is followed temporally by the re-establishment of nuclear functions such as DNA replication and transcription. We propose that the maternal transcription program is erased during oogenesis to generate a relatively naïve chromatin and the zygotic transcription program is rebuilt de novo after fertilization. This process is termed as the "erase-and-rebuild" process, which is used to reset the transcription program, and most likely other nuclear processes as well, from a maternal one to that of the embryo. We further show in the accompanying paper (Gao T, et al., Cell Res 2007; 17:135-150.) that the same strategy is also employed to reprogram transcriptional profiles in somatic cell nuclear transfer and parthenogenesis, suggesting that this model is universally applicable to all forms of transcriptional reprogramming during early embryogenesis. Displacement of CFs from chromatin also offers an explanation for the phenomenon of transcription silence during the maternal to zygotic transition.

Cell Research (2007) 17: 117-134. doi: 10.1038/cr.2007.1; published online 6 February 2007

Keywords: nuclear reprogramming, transcription, transcription silence, chromatin factors, embryogenesis, fertilization, oogenesis

\section{Introduction}

In mice, transcription ceases in the germinal vesicle (GV) oocytes [1-5]. Once ceased, it remains silent until zygotic genome activation (ZGA) takes place approximately 9-10 $\mathrm{h}$ after fertilization [6-9]. Between the two time points, from transcription cessation to resumption, the maternal

\footnotetext{
*These three authors contributed equally to this work Correspondence: Hui Z Sheng

Tel: +86-21-55570361; Fax: +86-21-55570017

E-mail: hzsheng2003@yahoo.com

www.nuclear-reprogramming.com.cn

Received 19 December 2006; revised 10 January 2007; accepted 12 January

2007; published online 6 February 2007
}

transcription profile is reprogrammed to that of the embryo. The molecular mechanism responsible for the reprogramming process underlying both normal development and somatic cell nuclear transfer (SCNT) is currently unknown [10-13]. Understanding the underlying mechanism is of central importance to developmental biology.

Three hypotheses have been proposed previously to explain transcription silence during embryogenesis: (1) transcription before middle blastula transition might be prevented by the rapid cell cycle during early Xenopus development [14-16]; (2) inhibitory factors in eggs might repress transcription [17-19]; and (3) deficiency [20] in or absence $[21,22]$ of critical transcriptional factors might cause transcription silence. These hypotheses, focusing mainly on events after fertilization, did not take into account 
events causing transcription cessation during oogenesis or spermatogenesis.

Several studies examined the expression levels and/or functional status of transcription factors or regulators during oogenesis, and found that nuclear concentrations of several transcription factors, such as SP1 and TATA-boxbinding protein (TBP), decrease during oocyte growth [23]. In addition, it was found that the component of the RNA polymerase II holoenzyme is dephosphorylated and translocated away from active sites [5, 24-27], and members of the polycomb group proteins (Ring1B and Rae28/Ph1) are dissociated from meiotic chromatin in GV oocytes [28]. Although it is conceived that these events could cause a deficient transcription in fully grown oocytes $[5,23,25,26]$, the scope and the data of the investigations were too limited to provide a sufficient explanation for the genome-wide transcription inactivation. Nor did these studies explain how these events contribute to reprogramming the gene expression profile. The issue is further complicated by the demonstration that subunits of the RNA polymerase I, upstream-binding factor (UBF), and proteins involved in rRNA processing and ribosome biogenesis remained associated with the nucleoli-like body when transcription had already been downregulated in surrounding nucleolus (SN) oocytes [29]. The primary mechanisms underlying transcription inactivation remains to be determined [3].

In eukaryotic cells, mRNA transcription begins with the assembly of the preinitiation complex on the promoter [30-33]. The pathway leading to gene transcription is also regulated in nearly every step by distinct multiprotein complexes, many of which bind to specific regulatory elements to regulate the generation of transcripts, and to modify nucleosomal and higher order chromatin structures. The combinatorial function of these chromatin factors (CFs) generates enormous specificities that define a given transcription profile. We hypothesized that switching from the maternal transcription profile to a zygotic one is accompanied by a global change in the CF-binding pattern on chromatin. One potential mechanism to achieve such a change efficiently is to erase the existing transcription program to generate a relatively naïve chromatin, onto which the zygotic program can be installed subsequently. To test this hypothesis, we examined dynamic changes in the relationship between chromatin and CFs in GV oocytes and early one-cell embryos.

Here we report that a broad range of CFs including essential transcription factors is dissociated from chromatin during the first meiotic cycle, which results in erasure of the maternal transcription program. Most CFs then re-associate with chromatin after fertilization subsequent to pronuclear formation. Re-association between chromatin and CFs is soon followed by the re-establishment of nuclear func- tions such as DNA replication [34, 35] and transcription [6-9]. Our results provide a new conceptual framework for transcription reprogramming. First, disassembly of the transcription apparatus and regulatory machinery is the primary cause responsible for transcription inactivation during oogenesis. Second, transcription reprogramming begins in oogenesis, well before fertilization. Oogenesis has an important, but not yet fully appreciated, function to erase the maternal transcription program so as to prepare the chromatin for zygotic development. Third, the maternal transcription program is transformed to the zygotic program through an across-the-board "erase-and-rebuild" process, instead of a restricted chromatin remodeling process, as implied by the existing data in the literature. Fourth, transcription silence at the transition point between the two generations is, for most part, a phenomenon intrinsically related to transcription reprogramming. It represents an inert status of the chromatin after the maternal transcription program is disassembled and before the zygotic equivalent is established. Fifth, the egg contains most, if not all, essential components for assembling the zygotic transcription program, and assembles it to the appropriate diploid chromatin of distinct origins. Sixth, the "erase-and-rebuild" process is also responsible for transcription reprogramming in SCNT and parthenogenesis [71]. It thus appears that a common strategy is used to build new life cycles in distinct forms of reproduction.

\section{Materials and Methods}

\section{Mouse strains}

Oocytes were obtained from F1 female mice between Kunming white and ICR (Center for Experimental Animals, Chinese Academy of Sciences, Shanghai, China). One-cell embryos were produced by crossing F1 female to Kunming male mice. To ensure that results obtained are not specific to a certain mouse strain, major observations in oocytes or one-cell embryos were verified on other mouse strains, either Kunming white or F1 from DBA and C57 (Beijing, China http://www.vitalriver.com.cn).

\section{Collection of GV oocytes and one-cell embryos}

To collect oocytes before ovulation, female F1 mice (5-6 weeks old) were injected with pregnant mare serum gonadotropin (PMSG, $10 \mathrm{IU})$. Forty eight hours later, ovaries were dissected and punctuated with 28-G needles in Hepes-buffered CZB [36], containing $3 \mathrm{mg} / \mathrm{ml}$ bovine serum albumin (BSA) to free oocytes. Three-isobutyl-1-methyl xanthine (IBMX, $0.2 \mathrm{mM}$ ) was added to the medium to inhibit GV breakdown. Oocytes were cultured in MEM $\alpha$ (Invitrogen, Carlsbad, CA, USA) supplemented with $3 \mathrm{mg} / \mathrm{ml} \mathrm{BSA}, 1 \mathrm{mg} / \mathrm{ml}$ Fetuin, $1 \mathrm{ng} / \mathrm{ml}$ EGF and $100 \mathrm{ng} / \mathrm{ml} \mathrm{FSH}$ at $37{ }^{\circ} \mathrm{C}, 5 \% \mathrm{CO}_{2}$ for $0.5,1,2$, or $6 \mathrm{~h}$, respectively.

To collect MII oocytes, F1 female mice were injected with 10 IU PMSG and followed $48 \mathrm{~h}$ later by $10 \mathrm{IU}$ hCG. Oocytes at the MII stage were obtained from oviducts $18-20 \mathrm{~h}$ post-hCG injection. Cumulus cells were removed in $0.5 \%$ hyaluronidase (Sigma). 
Table 1 Antibodies used in this study

\begin{tabular}{|c|c|c|c|c|}
\hline$\overline{\mathrm{CF}}$ & Company & Cat No. & Dilution & Protocol $^{1}$ \\
\hline$\overline{\mathrm{TBP}}$ & Santa Cruz Biotechnology Inc. & sc-204 & $1: 50$ & $\mathrm{C}$ \\
\hline TRF3 & Abgent, USA & & $1: 500$ & A \\
\hline TAF1 & Santa Cruz Biotechnology Inc. & sc-46649 & $1: 50$ & $\mathrm{C}$ \\
\hline TAF4 & BD Biotechnology Inc. & 612054 & $1: 50$ & $\mathrm{C}$ \\
\hline Pol II & COVANCE Biotechnology Inc. & MMS-126R & $1: 50$ & $\mathrm{C}$ \\
\hline Pol II & Santa Cruz Biotechnology Inc. & sc-5943 & $1: 50$ & $\mathrm{C}$ \\
\hline BRF1 & generated in house & & $1: 500$ & $\mathrm{C}$ \\
\hline $\mathrm{AP} 2 \alpha$ & Santa Cruz Biotechnology Inc. & sc-184 & $1: 50$ & $\mathrm{C}$ \\
\hline INI1 & Santa Cruz Biotechnology Inc. & sc-9751 & $1: 50$ & $\mathrm{C}$ \\
\hline YY1 & Santa Cruz Biotechnology Inc. & sc-281 & $1: 50$ & $\mathrm{C}$ \\
\hline HDAC1 & Santa Cruz Biotechnology Inc. & sc- 8410 & $1: 50$ & $\mathrm{C}$ \\
\hline HDAC2 & Upstate Biotechnology Inc. & $05-814$ & $1: 50$ & $\mathrm{C}$ \\
\hline HDAC2 & Santa Cruz Biotechnology Inc. & sc-6296 & $1: 50$ & $\mathrm{C}$ \\
\hline $\mathrm{MeCP} 2$ & Santa Cruz Biotechnology Inc. & sc-5755 & $1: 50$ & $\mathrm{C}$ \\
\hline MBD2a,b & Sigma Inc. & M7318 & $1: 50$ & $\mathrm{C}$ \\
\hline $\mathrm{HP} 1 \alpha$ & Santa Cruz Biotechnology Inc. & sc-28735 & $1: 50$ & $\mathrm{C}$ \\
\hline $\mathrm{HP} 1 \alpha$ & Upstate Biotechnology Inc. & 05-689 & $1: 50$ & $\mathrm{C}$ \\
\hline
\end{tabular}

${ }^{1}$ Optimized protocol(s) for immunochemical studies of oocytes and one-cell embryos using the antibody.

One-cell embryos were obtained from mated female mice $15-25 \mathrm{~h}$ post-hCG injection and cultured in $\mathrm{CZB}$ medium at $37{ }^{\circ} \mathrm{C}, 5 \% \mathrm{CO}_{2}$ to desired developmental stages.

\section{Nuclear transfer into GV oocytes or MII eggs}

A single NIH3T3 cell was inserted under the perivitelline space of a GV oocyte. The cell and oocyte were fused with two DC pulses (240 $\mathrm{V} / \mathrm{cm}, 20 \mu \mathrm{s}$, BTX Cell Manipulator 2001) in a solution composed of $0.3 \mathrm{M}$ sorbitol, $0.1 \mathrm{mM}$ calcium-acetate, $0.1 \mathrm{mM}$ magnesium acetate and $0.5 \mathrm{mg} / \mathrm{ml} \mathrm{FFA-BSA} \mathrm{(Sigma).} \mathrm{Immediately} \mathrm{after} \mathrm{fusion,}$ reconstructed GV oocytes were incubated in MEM containing IBMX for $1 \mathrm{~h}$ and stained with Hoechest 33342 (Sigma). Being in culture for 6 to $8 \mathrm{~h}$ ( $5 \mathrm{~h}$ before nuclear transfer (nt), $1 \mathrm{~h}$ after nt, plus the time for nt operation), most oocytes were already in partly-SN (pSN) or SN configurations $[2,3]$. Reconstructed oocytes were incubated in MEM $\alpha$ without IBMX for an additional 2 to 5 or $12 \mathrm{~h}$ to obtain oocytes at later meiotic stages. Nuclear transfer into intact MII eggs was carried out using an established method except that the enucleation step was omitted [37].

Classification of $G V$ oocytes and one-cell embryos

GV oocytes have been classified into the NSN type, pSN type, and

SN type using criteria established previously [1, 4, 5, 38-41].

One-cell embryos before pronuclear formation were classified according to meiotic stages of the maternal chromosomes and collectively called "early one-cell embryos". Embryos after pronuclear formation were classified into stages 1-5, according to Adenot et al. (1997) [35]. For convenience, one-cell embryos after pronuclear formation are collectively termed "later one-cell embryos".

\section{BrUTP incorporation}

The BrUTP incorporation assay was performed according to a published method [42]. 
C
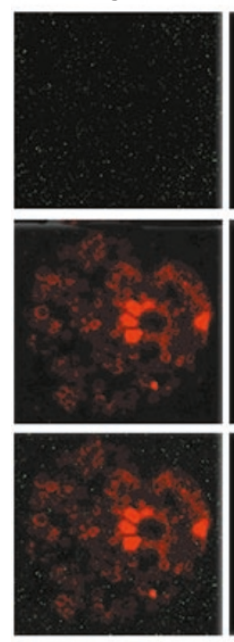

sNSN1
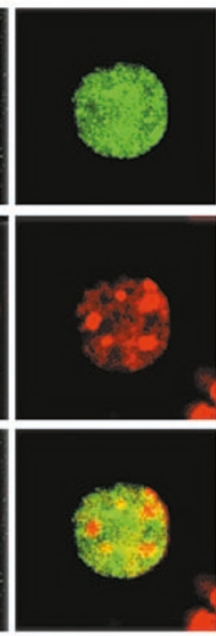

sNSN2
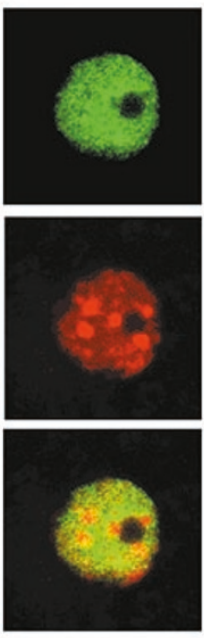

NSN
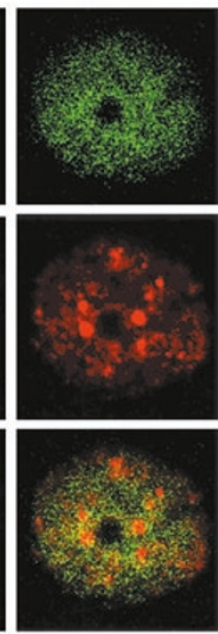

pSN
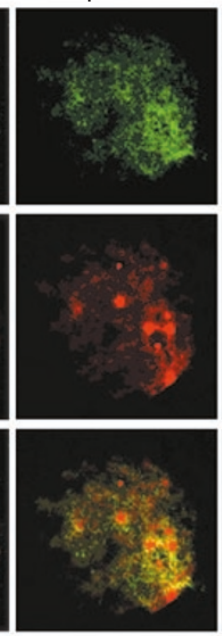

SN1
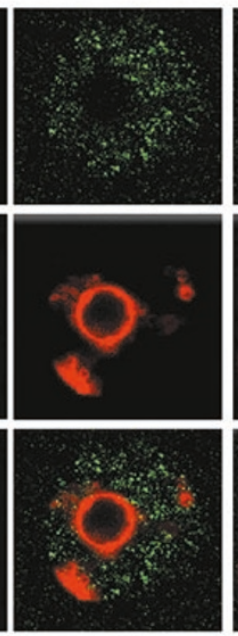

SN2

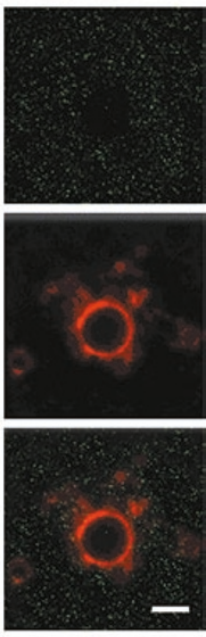

Figure 1 Transcription terminates gradually during chromatin condensation. BrUTP incorporation (green) was very active in smallsize GV oocytes ( $\mathrm{SNSN}$ ) and in a portion of NSN GV oocytes (NSN). It reduced during chromatin (red) condensation and vanished eventually in SN GV oocytes. C, control. The third row in each panel contained images merged from the top two rows. Bar=5 $\mu \mathrm{m}$.

\section{Sources of the antibodies}

The anti-TRF3 antibody and immunogen were generated as previously described [43]. BRF1 cDNA (full length; GenBank Accession No. BC058112) was PCR amplified, and expressed as BRF1-GST fusion protein. Affinity-purified peptides were used to immunize rabbits. The antibody detected only one band of the expected size in mouse embryo lysate in Western blot that could be blocked by purified immunogen (data not shown). All other antibodies and blocking peptides were purchased (Table 1).

\section{Indirect immune fluorescent assays}

Each antibody was tested against several protocols to optimize detection condition. A total of five different protocols was employed in this study. Table 1 lists protocol(s) optimized for each antibody. Images shown in the figures represent results using those protocol(s).

In protocol A, experimental samples (oocytes or embryos) were washed thrice in PBS, fixed for $1 \mathrm{~h}$ in 4\% paraformaldehyde (PFA) in PBS at RT, permeabilized for $10 \mathrm{~min}$ in $0.5 \%$ Triton X-100 in PBS, washed 3-6 times ( 5 min each) in 3\% BSA/PBS, and incubated overnight at $4{ }^{\circ} \mathrm{C}$ with primary antibodies, normal serum, or normal IgG (controls) diluted in 3\% BSA/PBS. On the second day, oocytes or embryos were washed thrice, incubated with CY3- or FITC-conjugated anti-rabbit/goat/mouse antibody diluted in 3\% BSA/PBS for $1 \mathrm{~h}$, washed thrice, counterstained with Hoechst 33342, mounted on glass slides in 50\% glycerine-mounting solution, and examined under a confocal microscope (Fluoview 1000, Olympus). In protocol B, samples were permeabilized in $0.5 \%$ Triton-100 in PBS for $10 \mathrm{~min}$ at RT, and fixed in $4 \%$ PFA in PBS at $4{ }^{\circ} \mathrm{C}$ overnight. In protocol $\mathrm{C}$, samples were fixed and permeabilized at the same time with $1 \%$ PFA and $0.2 \%$ Triton- 100 in PBS overnight at $4{ }^{\circ} \mathrm{C}$. In protocol $\mathrm{D}$, samples were fixed with methanol (pre-chilled in $-20{ }^{\circ} \mathrm{C}$ ) and permeabilized in $0.5 \%$ Triton X-100 in PBS for $10 \mathrm{~min}$ at RT. In protocol E, samples were fixed with $70 \%$ ethanol for $10 \mathrm{~min}$ at RT, rehydrated in $0.3 \% \mathrm{BSA} / \mathrm{PBS}$ for $10 \mathrm{~min}$. The rest of the procedure was the same as in protocol A.

\section{Specificity of immunochemical detection}

Specificity of antibody labeling was verified using the following procedures. (1) Normal goat or rabbit serum (Jackson Immunoresearch Laboratories), or normal mouse IgG (Sigma) were used as negative controls for each antibody and included in all experiments. NIH3T3 cells, which expressed most CFs in nuclei, were used as positive controls. In many cases, CFs were presented in embryos of one but not the other stage in the same batch, those embryos were used as additional positive or negative controls. (2) Blocking peptides were purchased or synthesized for 10 antibodies when the immunogene or its sequence was available.

\section{Blocking peptides}

Each antibody was incubated with the corresponding blocking peptide (at a concentration approximately 10 times of the antibody) for $1 \mathrm{~h}$ at RT before applying the antibody to samples. A positive control experiment was carried out in parallel using the same protocol without adding in the peptide.

\section{Construction of GFP-CF expression vectors}

Seven GFP-CF fusion proteins were constructed. These included GFP-TBP, GFP-TRF3, GFP-TFIIB, GFP-BRF1, GFP-HP1 $\beta$, GFP$\mathrm{MeCP} 2$, and GFP-Histone 2B (H2B). Data from immunochemical and biochemical analyses demonstrated that these fusion proteins behaved similarly to endogenous factors (www.nuclear-reprogramming.com.cn). Expression vectors were tranfected into NIH3T3 cells using lipofectamine 2000 (Invitrogen, CA, USA). Only cells with very weak fluorescence were used for nuclear transfer.

\section{Cell culture and plasmid transfection}

Murine NIH3T3 embryo fibroblasts and human HeLa cells were obtained from the Cell Bank of the Chinese Academy of Sciences 
A

C

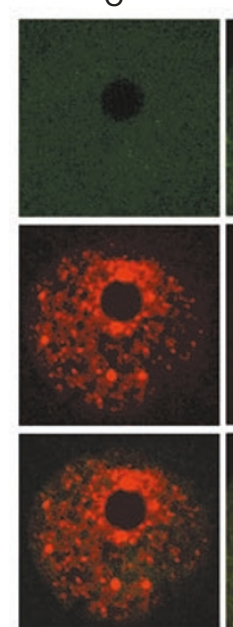

C

B

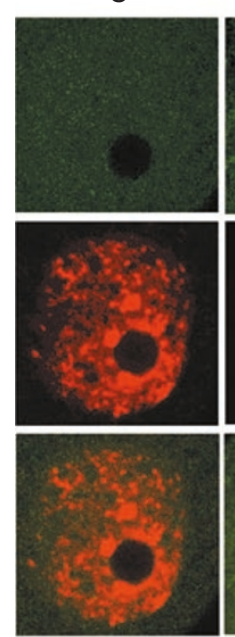

NSN
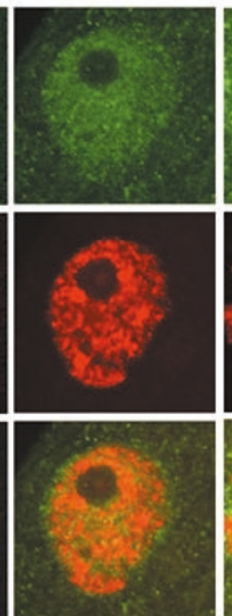

NSN
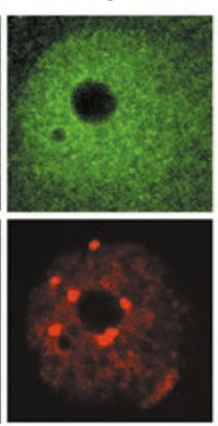

C
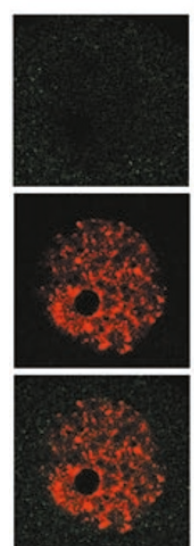

pSN
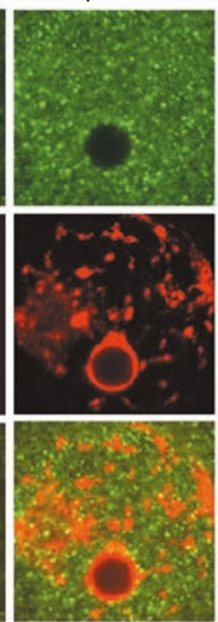

pSN
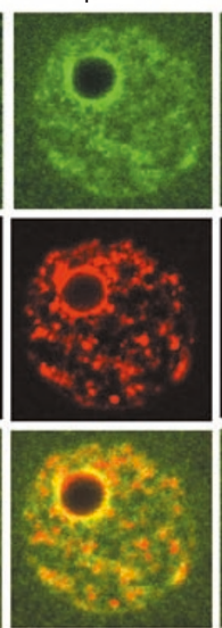

SN
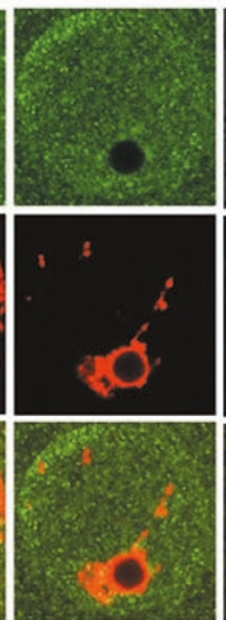

SN
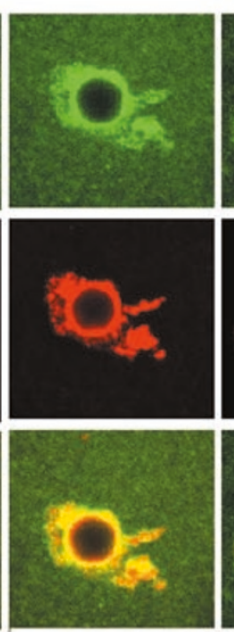

SN1

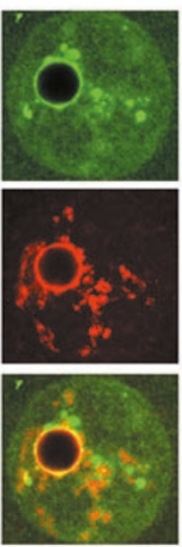

SN2
Dia
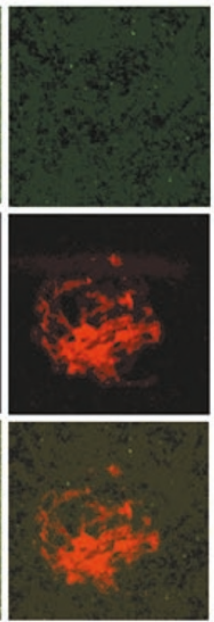

Dia
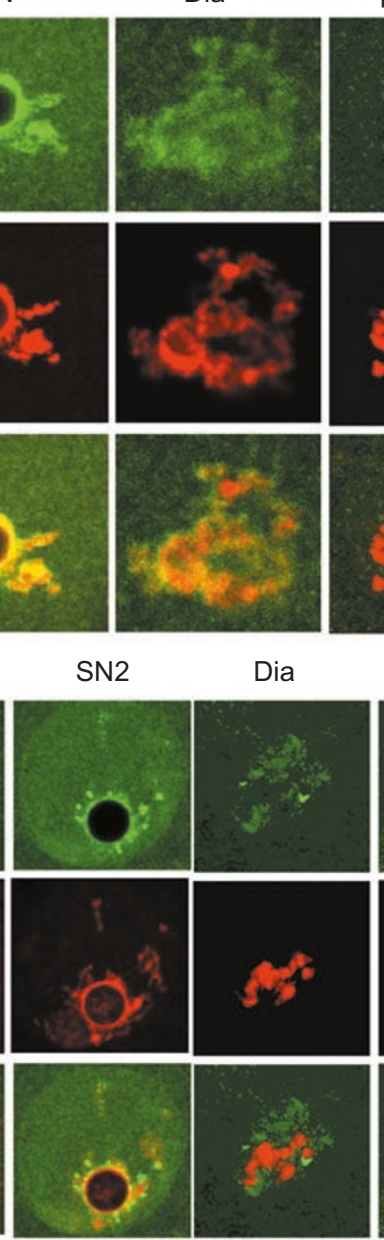

pro-MI
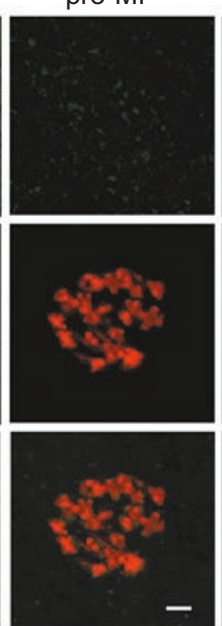

pro-MI

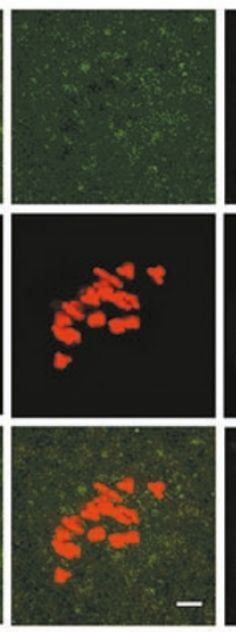

pro-MI

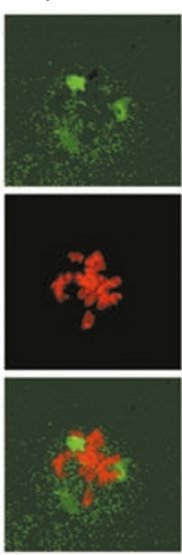

$\mathrm{MI}$ *
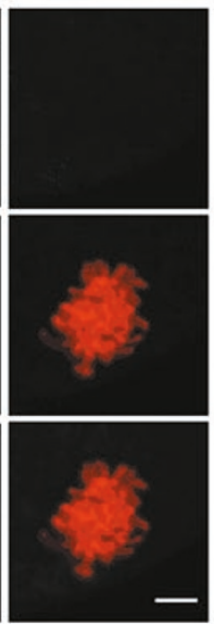

$\mathrm{MI}$ *

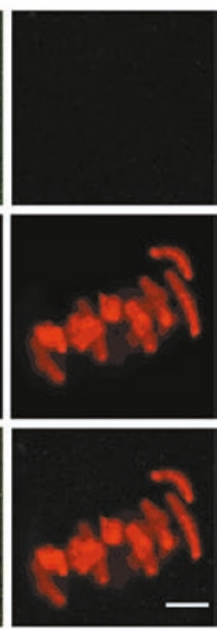

MI

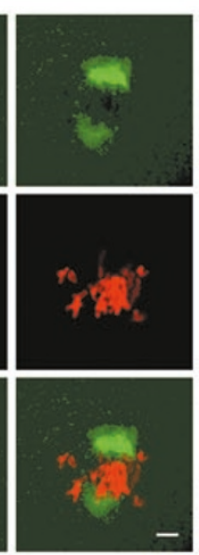

Figure 2 Dissociation of GTFs from chromatin during chromatin condensation. TBP (A), TAF1 (B), and TFIIB (C) (green) were detected in NSN GV nuclei as signals distributed to the entire nuclear area. During chromatin (red) condensation, most molecules of TBP and TFIIB did not co-localize or migrate together with chromatin while most TAF1 molecules remained bound to chromatin before GVBD. TAF1 became dissociated from chromatin before MI. C, control. The third row in each panel contained images merged from the top two rows. Bar=5 and $10 \mu \mathrm{m}$ in low and high (*) magnitude, respectively. See Supplementary information I for more details with these CFs. 
Table 2 Subcellular distribution of CFs in meiotic oocytes

\begin{tabular}{|c|c|c|c|c|c|c|}
\hline & $\mathrm{NSN}^{1}$ & $\mathrm{pSN}^{2}$ & $\mathrm{SN}^{2}$ & $\mathrm{Dia}^{2}$ & pro-MI ${ }^{2}$ & $\mathrm{MI}^{2}$ \\
\hline$\overline{\mathrm{TBP}}$ & $+27^{3}$ & -59 & -82 & -22 & -31 & -8 \\
\hline TRF3 & +5 & -6 & -14 & -2 & -3 & -2 \\
\hline TAF1 & +37 & +64 & +98 & +18 & $+24 /-20$ & -32 \\
\hline TAF4 & +18 & -17 & -42 & -20 & -15 & -11 \\
\hline TFIIA & +20 & -26 & -49 & -38 & -66 & -12 \\
\hline TFIIB & +29 & -24 & -70 & -20 & -22 & -11 \\
\hline BRF1 & +24 & -25 & -63 & -12 & -2 & -4 \\
\hline $\mathrm{AP} 2 \alpha$ & +8 & -12 & -15 & -18 & -8 & -7 \\
\hline Pol II & +16 & -18 & -53 & -5 & -40 & -17 \\
\hline BRG1 & +27 & \pm 21 & \pm 64 & $+5 /-17$ & -25 & -18 \\
\hline SRG3 & +15 & +31 & +89 & -10 & -28 & -13 \\
\hline Inl1 & +20 & \pm 21 & \pm 52 & $+1 /-17$ & -41 & -12 \\
\hline YY1 & +7 & -9 & -24 & -25 & -35 & -12 \\
\hline HP1 $1 \alpha$ & +8 & +13 & +25 & +9 & +15 & +14 \\
\hline HP $1 \beta$ & +55 & +63 & +199 & $+6 /-12$ & $+12 /-11$ & +9 \\
\hline $\mathrm{MeCP} 2$ & +13 & \pm 19 & -24 & -25 & -43 & -21 \\
\hline MBD2 & +42 & -53 & -138 & -18 & -23 & -12 \\
\hline HDAC1 & +25 & +21 & +49 & $+3 /-14$ & -36 & -13 \\
\hline HDAC2 & +21 & +13 & +43 & $+3 /-16$ & $+4 /-31$ & -29 \\
\hline TopoII $\alpha$ & +15 & +30 & +23 & +26 & +30 & +21 \\
\hline TopoII $\beta$ & +23 & +37 & +83 & +12 & +23 & +32 \\
\hline Ac-H4 & +39 & +20 & +91 & +28 & \pm 11 & \pm 9 \\
\hline
\end{tabular}

${ }^{1}$ Antibody signals are either higher than $(+)$ or the same as $(-)$ negative controls.

${ }^{2}$ Antibody signals on chromatin are higher $(+)$ or lower $(-)$ than the cytoplasm, \pm the amount bound to chromatin was significantly decreased but still detectable.

${ }^{3}$ The numbers of embryos with the phenotype (all embryos examined are included in the table, i.e., when only one number is in the column, $100 \%$ of the embryos had the particular phenotype).

(Shanghai, China), and mouse embryonic stem cell line (D3, ATCC) was cultured according to the protocols by the supplier.

Cumulus cells were obtained from the ovary and used directly for SCNT experiments or in immunochemical studies.

\section{Data collection using confocal microscope}

Samples were scanned using an Olympus laser-scanning confocal microscope equipped with a $\mathrm{CO}_{2}$ and thermal controller system. To scan living cells, laser strength and scanning duration were reduced to minimize damage to cells.

\section{Data presentation}

The "Results" section in the main text summarizes the major findings of the study. Detailed descriptions of subcellular distributions of each CF are presented in the expanded figure legend under each figure. The expanded legends explain procedures used to optimize detection conditions and verify specificity of the labeling. Results from previous publications were also discussed when such data were available.

Abbreviations used in figures: For GV oocytes: sNSN: small-sized oocytes with the NSN configuration; NSN: the NSN configuration; pSN: the partial SN configuration; SN: the SN configuration; Dia: diakinesis; pro-MI: prometaphase I; MI, metaphase I; MII, metaphase II. For fertilized embryos: Ana, 1-cell embryos at anaphase; PN1, pronuclear stage 1; PN2/3, pronuclear stage 2 and 3; PN4/5, pronuclear stage 4 and 5; 2-cell, embryos at the 2-cell stage. For nt-embryos: $0 \mathrm{~h}$, within 15 min after nt; $2 \mathrm{~h}: 2 \mathrm{~h}$ after nt; A1 h, A4 h, A12 h are 1, 4 , and $12 \mathrm{~h}$ after activation; 2-cell, nt-embryos at the 2-cell stage; PPN: pseudo-pronuclei; FPN, female pronuclei.

\section{Results}

Transcription cessation is accompanied by an across-theboard removal of transcription factors and regulators from chromatin during the first meiotic cycle

In mice, transcription silence is temporally correlated with the process when chromatin changes from a nonsurrounding nucleolus (NSN) configuration to an SN configuration [38-41]. Transcription activity is high in NSN oocytes, decreases during chromatin condensation, and ceases in SN oocytes [1-5]. This was verified independently (Figure 1).

We first wanted to know whether the transcription machinery was still intact on the chromatin during the period when transcription activity was vanishing. In NSN nuclei, transcription was active (Figure 1) and the eight general transcription factors (GTFs) examined (TBP, TRF3, TAF1, TAF4, TFIIA, TFIIB, Pol II, and BRF1) were present (NSN; Figure 2 and Supplementary information I-1 to I8). Therefore, the transcription machinery must still be on the chromatin and functioning at this stage. In partial SN $(\mathrm{pSN})$ and $\mathrm{SN}$ nuclei, chromatin gradually shrunk. Movement of the chromatin provided an opportunity to examine the relationship between GTFs and chromatin. As shown in Figure 2, TAF1 was co-localized with DNA during chromatin condensation. After germinal vesicle breakdown (GVBD), it was detected on chromatin/chromosomes in all diakinesis oocytes, and in approximately half of the proMI oocytes, but was absent in MI oocytes (Table 2). These results indicated that TAF1 was associated with the DNA template during chromatin condensation and was dissociated progressively after GVBD. In contrast to TAF1, most molecules of TBP, TRF3, TAF4, TFIIA, TFIIB, BRF1, and Pol II remained distributed throughout the nucleoplasm and were not co-localized with chromatin during chromatin condensation (Figure 2, Supplementary information I-1 to I-8, and Table 2). This indicates that a large proportion of TBP, TRF3, TAF4, TFIIA, TFIIB, Pol II, and BRF1 molecules had already dissociated from chromatin when chromatin condensation became visible. After GVBD, none of the seven GTFs were detected on the chromatin/chro- 
A

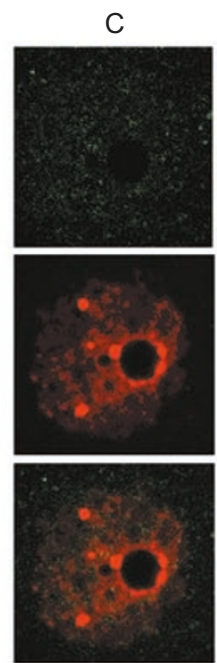

C

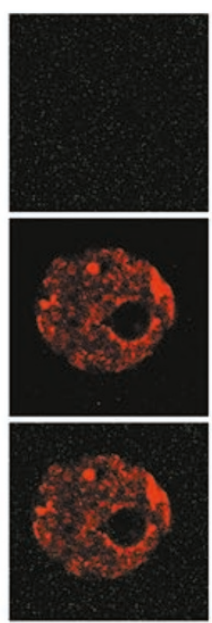

C

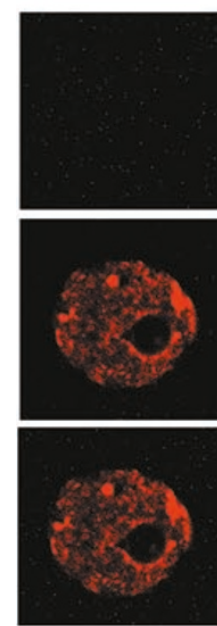

NSN
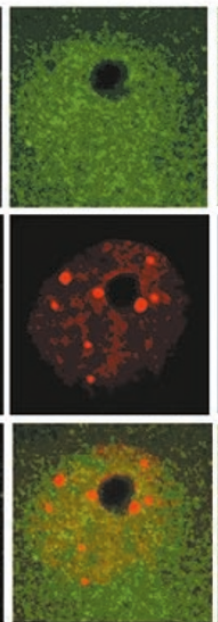

NSN
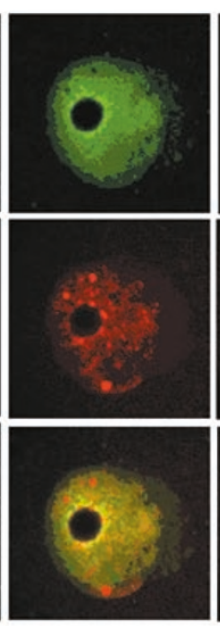

NSN
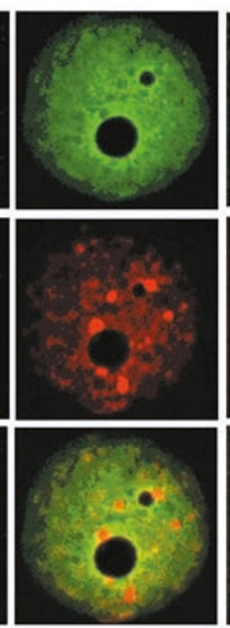

pSN
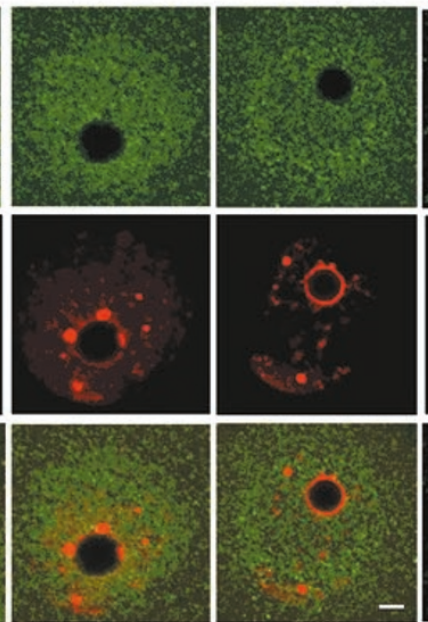

pSN

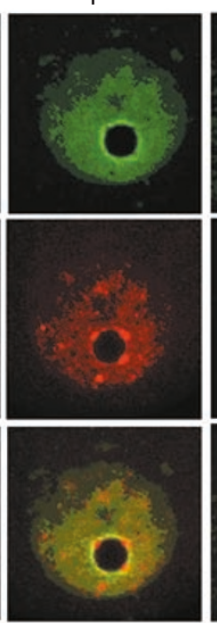

pSN

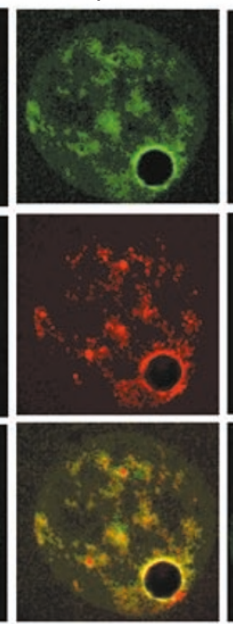

SN

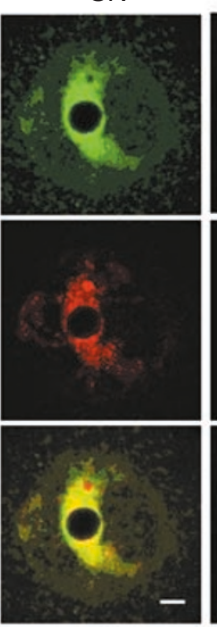

SN
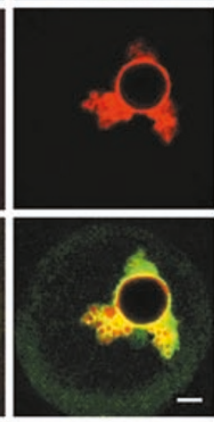

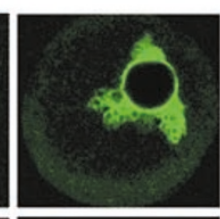

Dia *
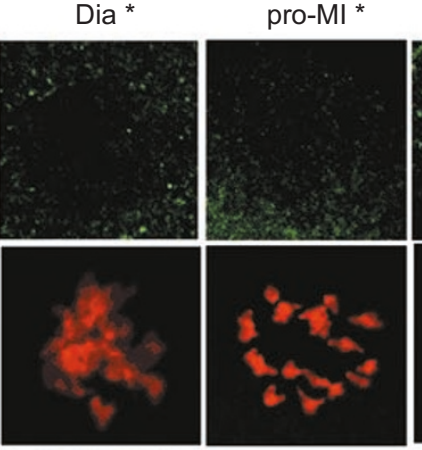

$\mathrm{Ml}$ *
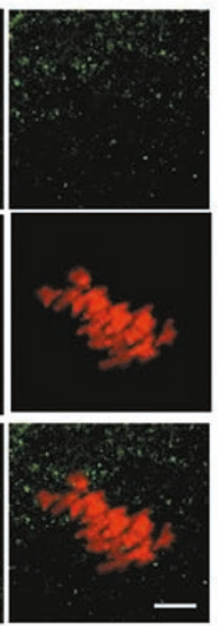

Dia *

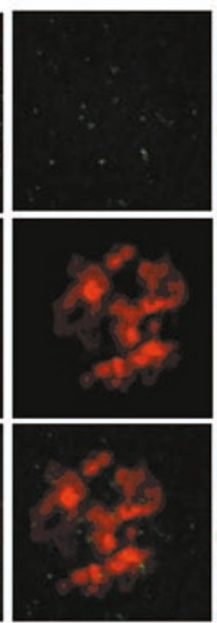

Dia

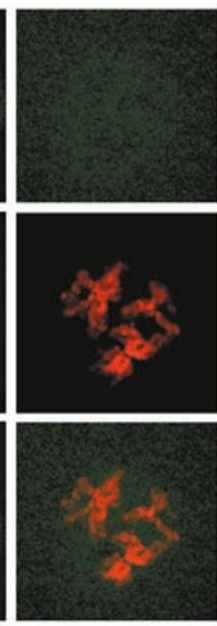

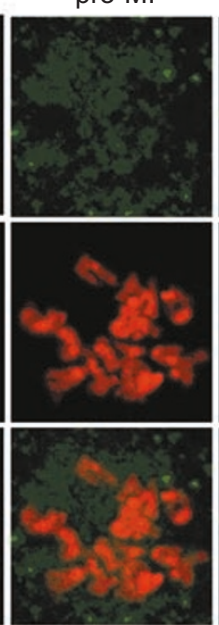

pro-MI

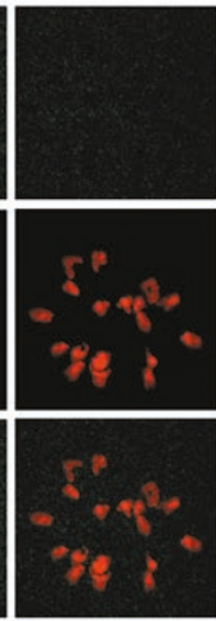

$\mathrm{Ml}$ *

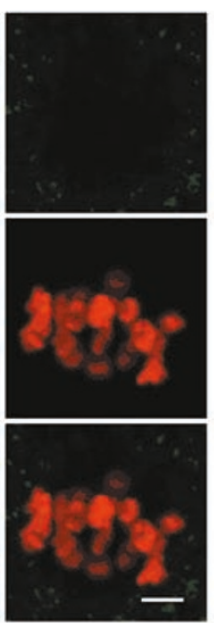

MI

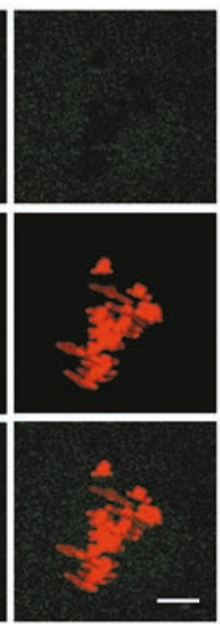

Figure 3 Dissociation of transcription activator and remodeling factors from chromatin during chromatin condensation. AP2 $\alpha$ (A), BRG1 (B), and SRG3 (C) (green) were detected in NSN GV nuclei as signals distributed to the entire nuclear area. During chromatin (red) condensation, most BRG1 and SRG3 molecules were co-localized and migrated together with chromatin while AP2 $\alpha$ remained distributed throughout the nucleus. All three CFs were dissociated from chromatin after GVBD. C, control. The third row in each panel contained images merged from the top two rows. Bar $=5$ and $10 \mu \mathrm{m}$ in low and high (*) magnitude, respectively. See Supplementary information I for more details with these CFs. 
mosome (Figure 2, Supplementary information I-1 to I-8, and Table 2). Results from our immunochemical studies indicated that most molecules of the eight GTFs examined dissociated from chromatin before metaphase I.

The path leading to transcription is regulated extensively by distinct multiprotein complexes. Sequence-specific transcription factors contact the core transcription machinery and activate transcription [44]. ATP-dependent remodeling factors use ATP hydrolysis to increase the accessibility of nucleosomal DNA, which is a fundamental requirement for several steps in transcription $[45,46]$. We examined the binding status of selective members of these complexes during meiosis. These included AP2 $\alpha$ (transcription factor), BRG1 (Smarca4), SRG3 (Smarcc1), INI1 (Smarcb1) (remodeling factors), and YY1 (a polycomb group protein that acts as a transcriptional repressor, an activator, or an initiator element-binding protein [47]). As shown in Figure 3, Supplementary information I-9 to I-13, and Table 2 , the five CFs were distributed throughout nuclei in NSN GV oocytes. During chromatin condensation, while most molecules of AP $2 \alpha$, INI1, and YY1 were dissociated from chromatin, BRG1 and SRG3 were retained on chromatin. After GVBD, none of the five CFs were detected on chromatin/chromosomes. These results indicated that a range of transcription regulators was also dissociated from chromatin before metaphase I.

\section{A cohort of structural proteins remained bound to chro- matin during meiosis}

Next, we extended the study to factors that are essential for establishing, maintaining, and regulating chromatin structure and function. These included HDAC1, HDAC2, MeCP2, MBD2, HP1 $\alpha$, HP1 $\beta$, TOPOII $\alpha$, and TOPOII $\beta$. The opposite actions of histone acetytransferase and histone deacetylase create specific patterns of hyper- and hypo-acetylation that correlate with transcription activation and repression, respectively $[48,72]$. Methyl-binding proteins bind specifically to methylated DNA and mediate the biological roles of methyl-CpG [49]. Topoisozymerases and heterochromatin-binding proteins are required for maintaining the normal chromatin structure [50, 51]. Some of them also play critical roles in transcription [52] and DNA replication [50, 53]. Acetylated histone 4 (AcH4) was used as a positive control. Again, all of these eight $\mathrm{CFs}$, together with the positive control, were detected in NSN nuclei. During chromatin condensation, HDAC1, HDAC2, MeCP2, MBD2, and TOPOII $\beta$ were dissociated from chromatin, in most cases partially, while a substantial amount of HP1 $\alpha, \mathrm{HP} 1 \beta$, TOPOII $\alpha$, and $\mathrm{AcH} 4$ was bound persistently. After GVBD, HDAC1, HDAC2, and MBD2 were not detected on chromosomes, a weak signal of MeCP2 was seen on chromatin in diakinesis but not in later stage oocytes. The amount of HP1 $\beta$ and TOPOII $\beta$ bound to chromatin dropped to barely detectable levels in later diakinesis, while their signals became more visible in some regions of chromosomes in the prometaphase and metaphase I. A substantial amount of HP1 $\alpha$ and TOPOII $\alpha$ remained on chromatin/chromosomes in all meiotic stages and so did AcH4 (Figure 4, Supplementary information I-14 to I-22, and Table 2). In summary, while HDAC1, HDAC2, MeCP2, and MBD2 were dissociated from DNA for a prolonged time, HP1 $\beta$ and TOPOII $\beta$ were dissociated from chromatin for only a short period of time, and a varying amount of $\mathrm{HP} 1 \alpha$, TOPOII $\alpha$, and $\mathrm{AcH} 4$ was bound persistently to chromatin/chromosomes. This was consistent with the fact that heterochromatin-binding factors, topoisomerases, and histones were essential for maintaining the chromatin structure. Even so, the binding patterns of some CFs in this group had changed visibly, implying that chromatin-binding sites of these CFs could have been reorganized during meiosis. HDAC1, HDAC2, MeCP2, and MBD2 are directly involved in transcription regulation, and they appear to be dissociated from chromatin for a prolonged time. Blocking peptides were available for 11 of the used antibodies. Labeling by these antibodies could be eliminated respectively by specific peptides corresponding to each CFs (Supplementary information I-23).

Therefore, within the sensitive range of immunochemistry, we detected a global dissociation of CFs from chromatin. Our data indicated that most molecules of TBP, TRF3, TAF1, TAF4, TFIIA, TFIIB, BRF1, Pol II, AP2 $\alpha$, BRG1, SRG3, INI1, YY1, MeCP2, MBD2, HDAC1, HDAC2, HP1 $\beta$, and TOPOII $\beta$ were dissociated from chromatin during the first meiotic cycle, implying that the maternal transcription machinery and regulatory mechanism are disassembled.

\section{Exogenous CFs follow the fate of endogenous $C F$ s during oogenesis}

To confirm independently the results from our immunochemical assays, we used GFP-CF fusion proteins to trace the subcellular distribution of CFs in GV oocytes. The fusion protein could be clearly visualized in all phases of mitosis [54, 55]. Vectors expressing GFP-coupled TBP, TRF3, TFIIB, BRF1, HP1 $\beta$, MeCP2, and H2B were constructed and transfected to NIH3T3 cells. Individual 3T3 cells expressing one of the seven fusion proteins were transplanted into oocytes. Within minutes, fusion proteins in transplanted nuclei began to migrate into GV nuclei (Supplementary information II-1). GFP-coupled HP1 $\beta$, $\mathrm{MeCP} 2$, and $\mathrm{H} 2 \mathrm{~B}$ targeted the nuclei and were bound strictly to chromatin, whereas GFP-coupled TBP, TRF3, TFIIB, and BRF1 targeted the nuclei, and were distributed evenly throughout the nucleus. The latter did not co-local- 
A
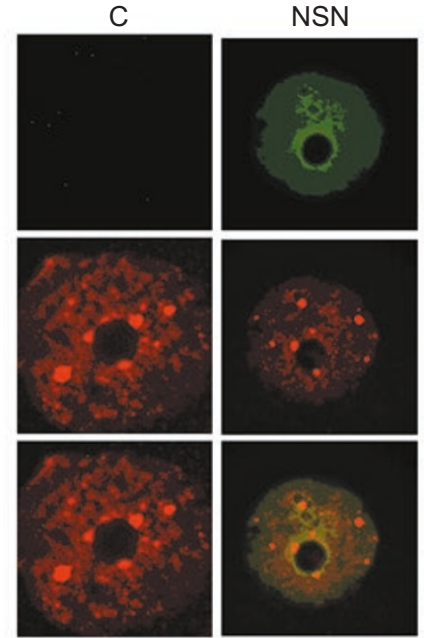

B

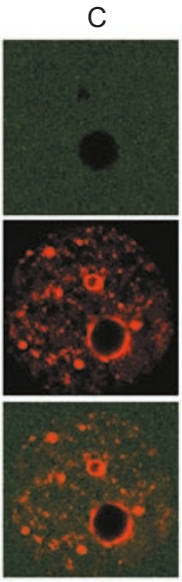

C
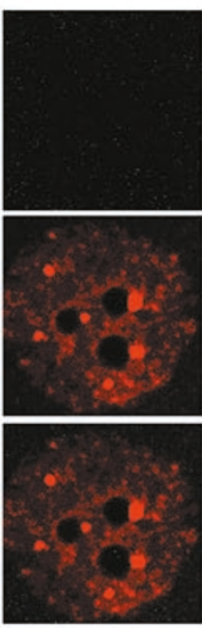

\begin{abstract}
NSN
\end{abstract}
NSN

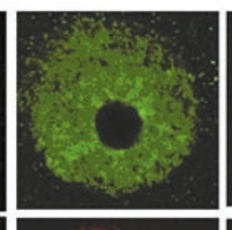

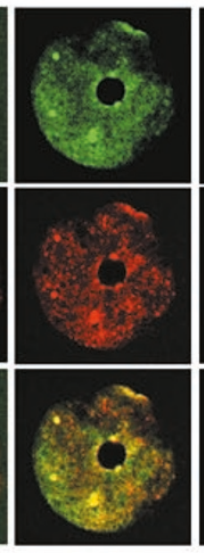

pSN
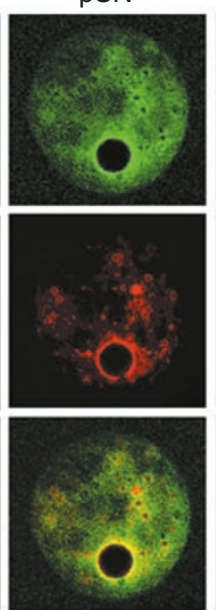

SN

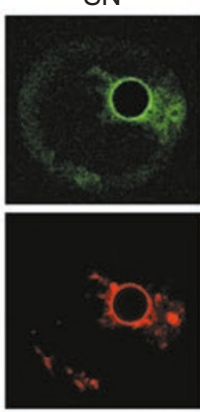

Dia *
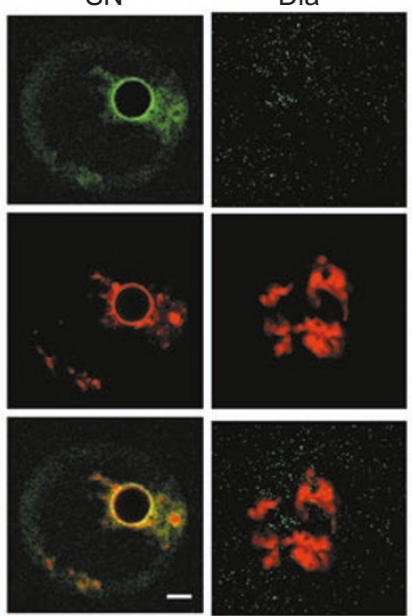

pro-Ml *
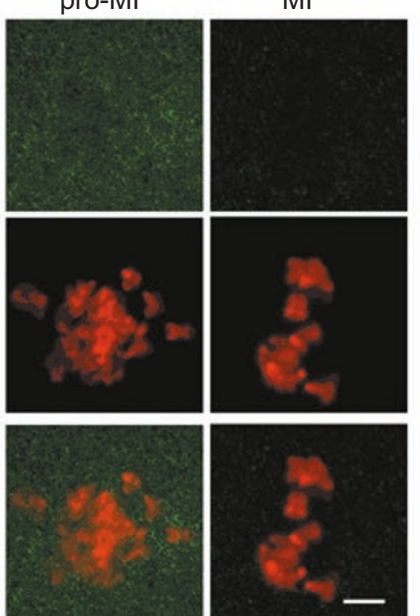

pSN
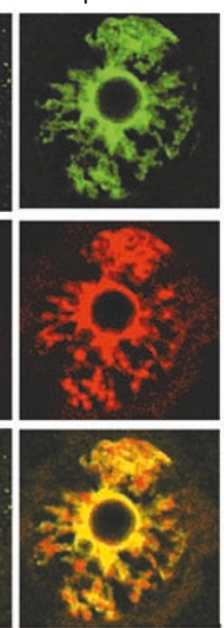

SN

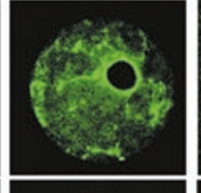

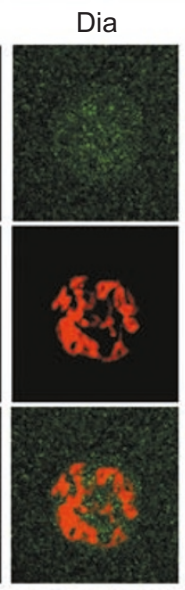

SN
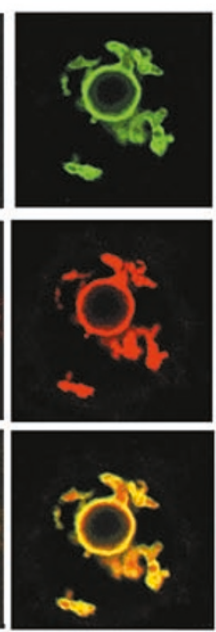

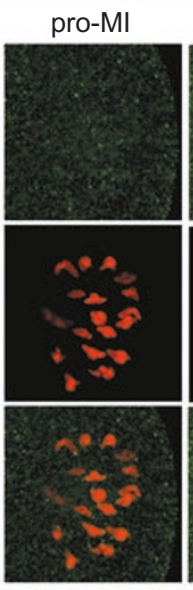

Dia
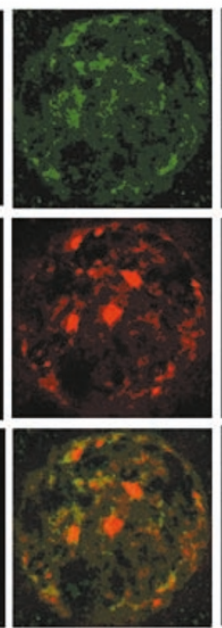

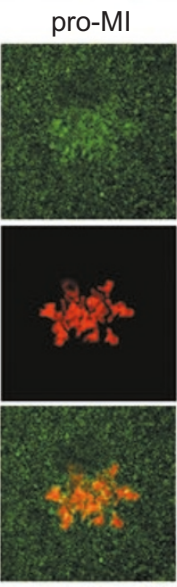

pro-MI
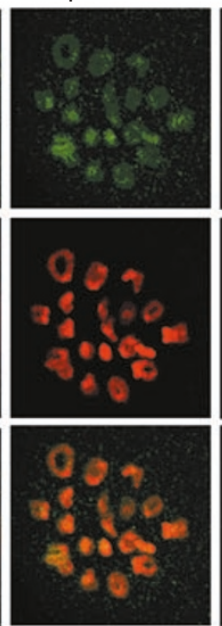

$\mathrm{MI}$

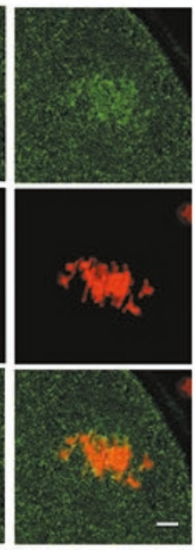

MI

Figure 4 Subcellular distribution of chromatin structural proteins and modifiers in meiotic oocytes. HDAC2 (A, green) was detected on chromatin before but not after GVBD. HP1 $\beta$ (B, green) was either spreading evenly in the nucleoplasm or concentrated in dotlooking structures in NSN oocytes. It co-localized with chromatin (red) during chromatin condensation. After GVBD, HP1 $\beta$ was not detected on chromatin/chromosome in later diakinesis and earlier Pro-M1, but was detected again in later pro-MI and MI phases, appearing more concentrated in the centromeres. (C) Ac-H4 was detected on chromatin/chromosome in oocytes of all meiotic phases. These results demonstrated that HDAC2 was dissociated from chromatin before GVBD, HP $1 \beta$ became dissociated from chromatin after GVBD, and histone 4 remained associated with chromatin/chromosomes in all stages. C, control. The third row in each panel represents images merged from the top two rows. Bar $=5$ or $10 \mu \mathrm{m}$ in low and high $\left(^{*}\right)$ magnitude, respectively. See Supplementary information I for more details with these CFs. 
A

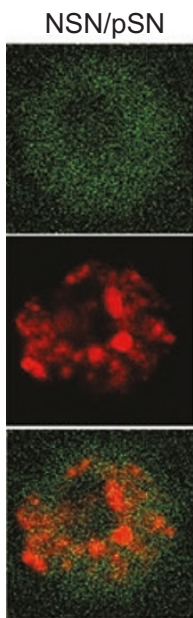

B

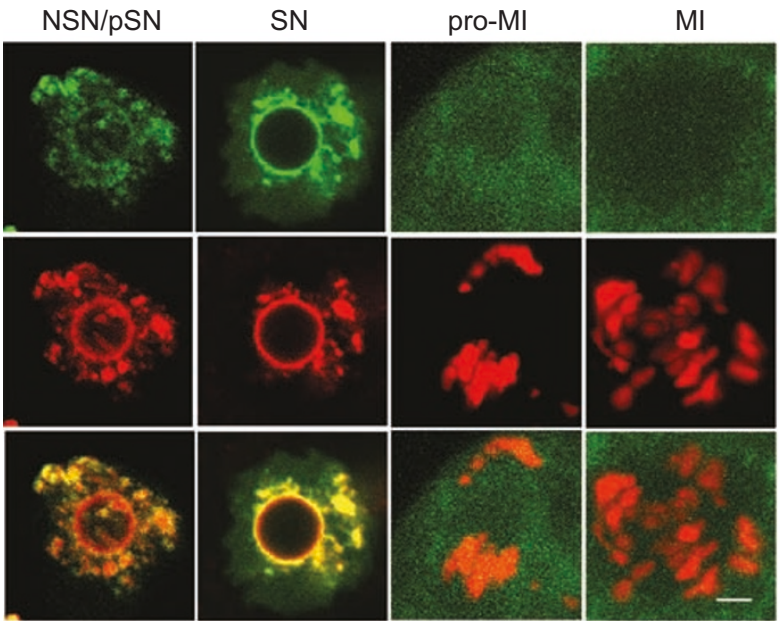

C
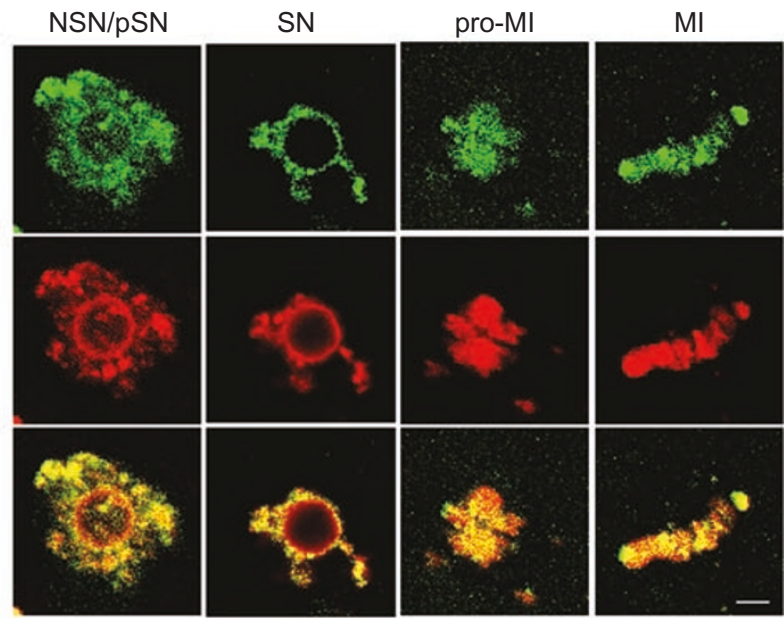

Figure 5 GFP-TBP, GFP-HP1 $\beta$, and GFP-H2B targeted to different subnuclear domains during chromatin condensation. All three GFPCFs (green) moved from the NIH3T3 nuclei (indicated by an arrow head if it was in the field) into GV nuclei within seconds to minutes after transplantation. During chromatin condensation, GFP-TBP(A) distributed evenly throughout GV nuclei, while HP1 $\beta$ (B) and H2B (C) targeted to chromatin (red). After GVBD, both GFP-TBP and GFP-HP1 $\beta$ redistributed to the cytoplasm of oocytes. In contrast, GFP-H2B remained on chromatin/chromosomes in all meiotic phases. The third rows are overlaid images of the top two rows. Bar $=5 \mu \mathrm{m}$. See Supplementary iniformation II for more details with these CFs.

ize or move together with chromatin. After GVBD, TBP, TRF3, TFIIB, BRF1, and HP1 $\beta$ were not associated with chromatin, while $\mathrm{MeCP} 2$ and $\mathrm{H} 2 \mathrm{~B}$ remained bound (Figure 5 , Supplementary information II and Table 3). These results indicated that the environment in $\mathrm{pSN}$ or SN GV nuclei promoted association between chromatin and HP1 $\beta, \mathrm{MeCP} 2$, and $\mathrm{H} 2 \mathrm{~B}$; but not TBP, TRF3, TFIIB, and BRF1. Thus, data from the fusion protein study are consistent overall with results from antibody staining except in two circumstances. First, MeCP2 was detected on the chromatin/chromosome in the later meiotic phases in the fusion protein assay but not in the immunochemical assay. It is possible that a low amount of MeCP2 may retain on chromatin, which could be detected by the fusion protein assay but not by the immunochemistry assay (see Supplementary information II-7 for more discussion). Second, a minute amount of HP1 $\beta$ was seen on the centromeres in prometaphase and metaphase chromosomes by the immunochemical assay but not by the fusion protein assay. The reason for this discrepancy is not clear at this time. 
A

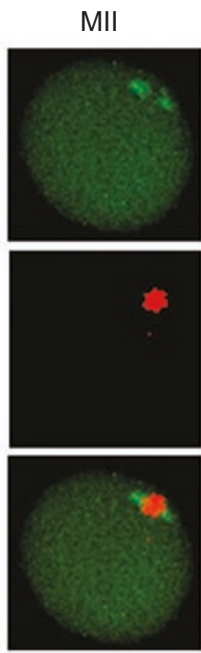

MII

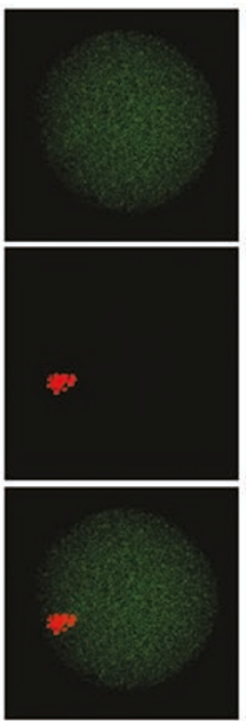

C

MII
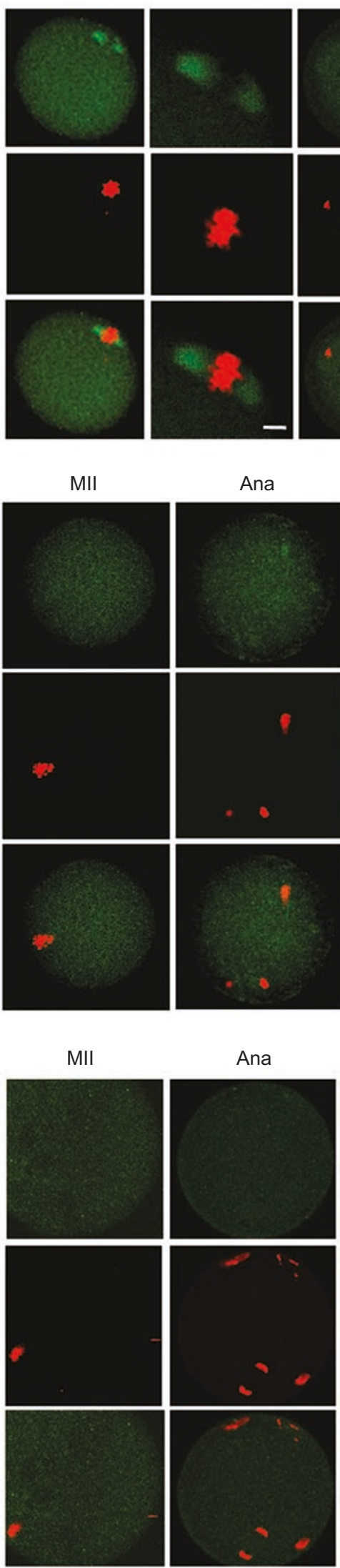

B

Ana

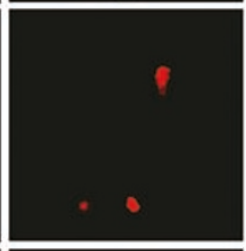

5
Ana

PN1
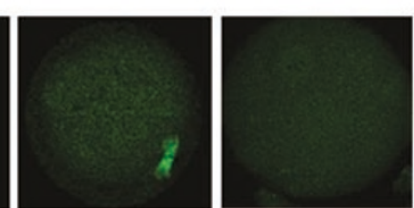

PN2/3

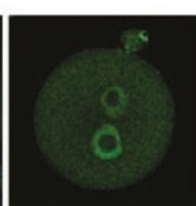

PN4/5

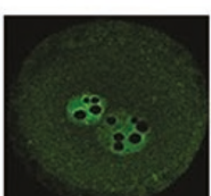

2-cell
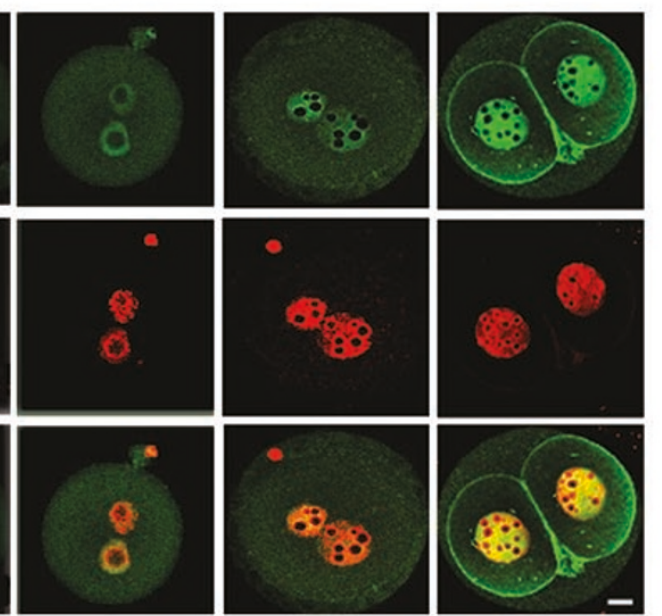

PN1

PN2/3
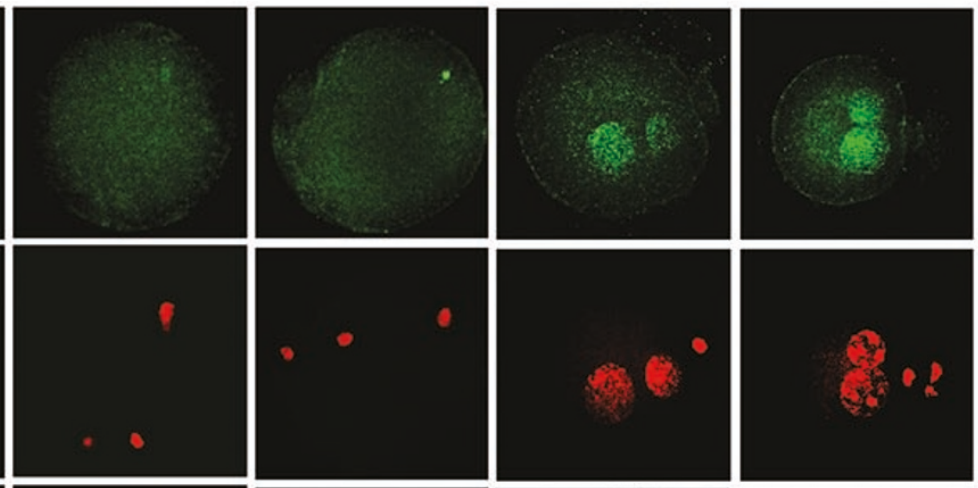

•.

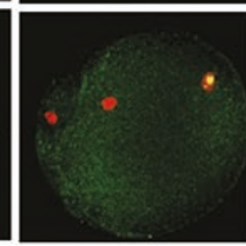

PN1

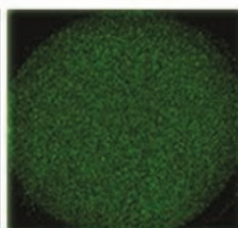

PN2/3
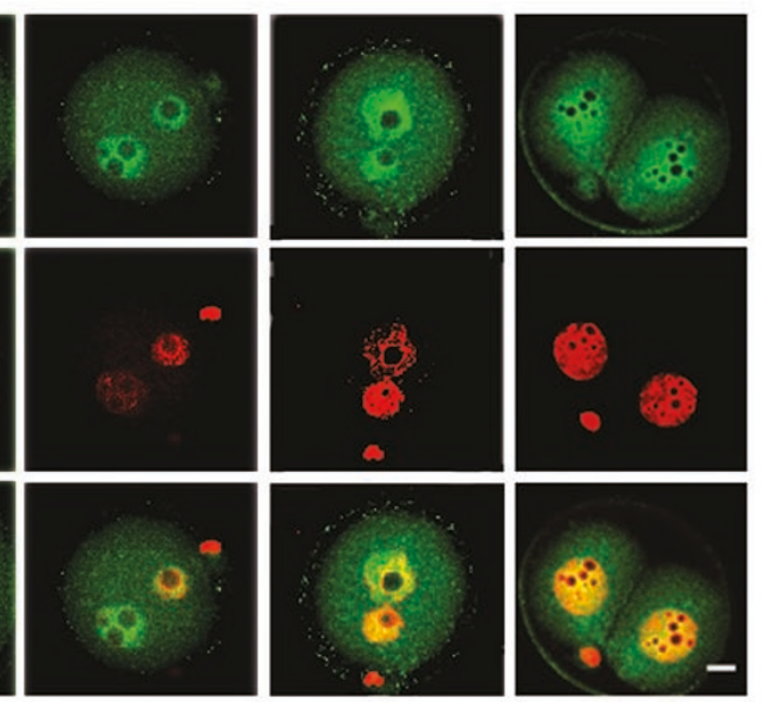
D
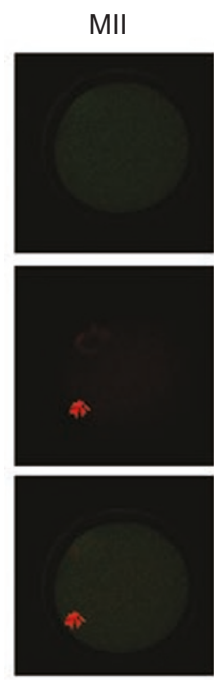

E

MII
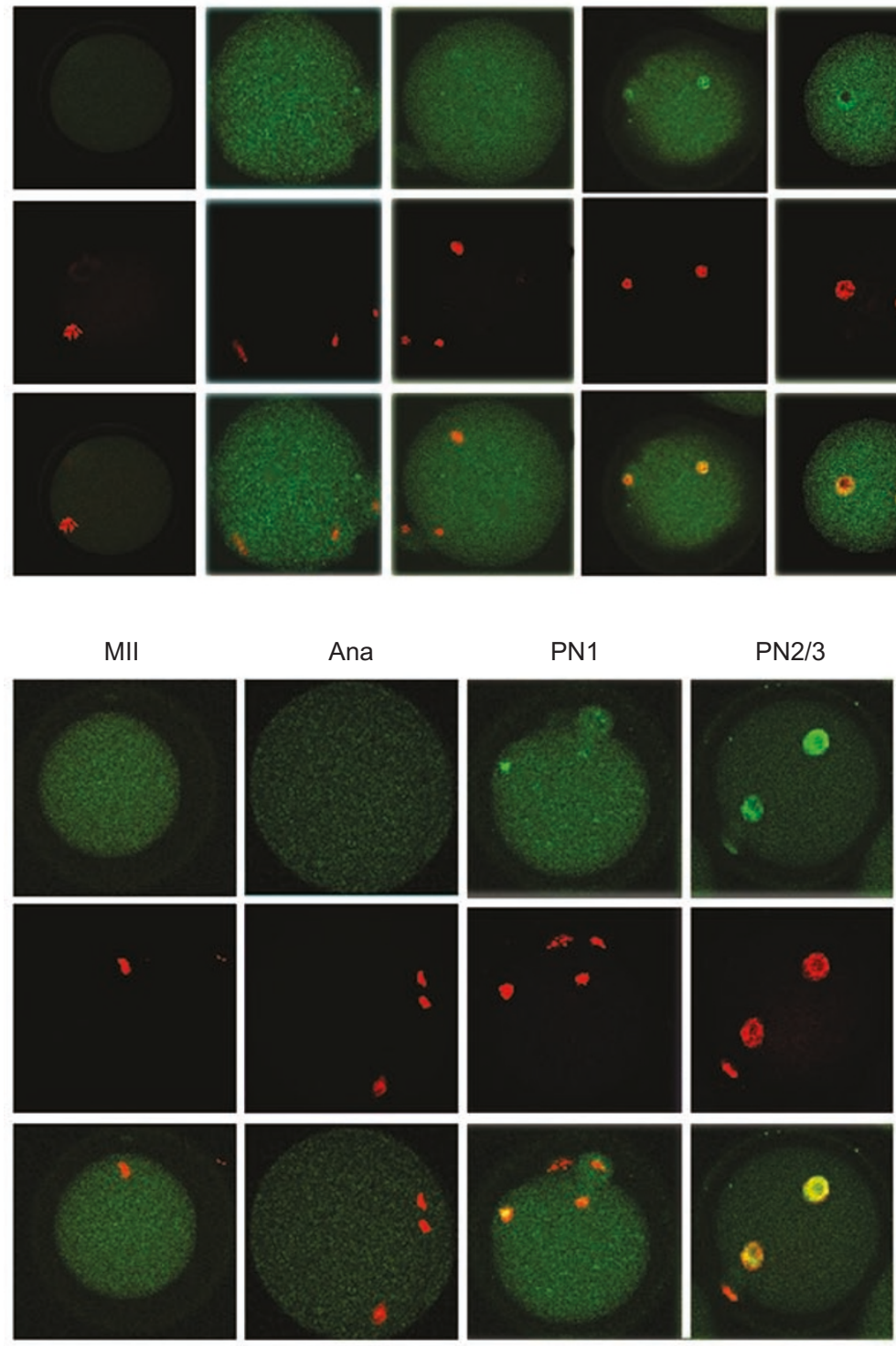

F
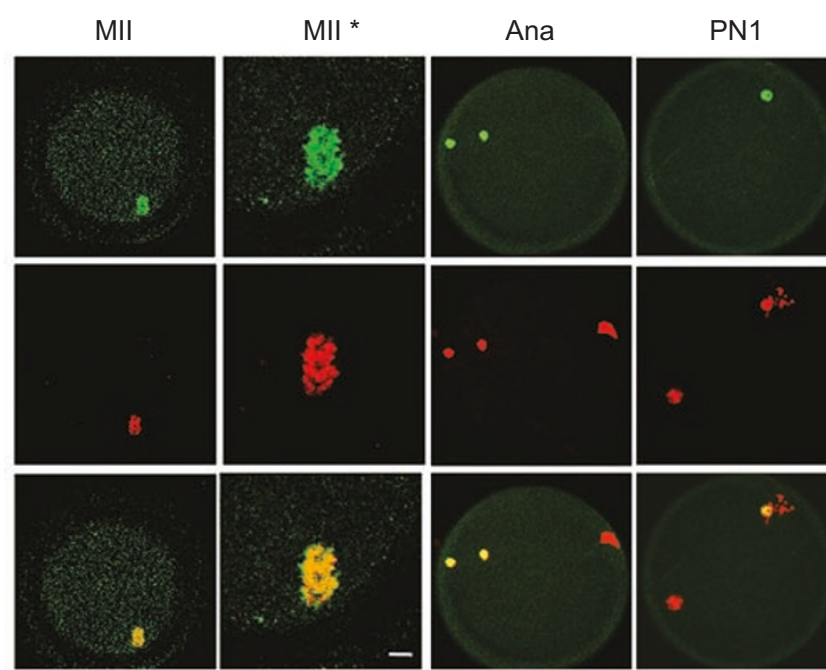

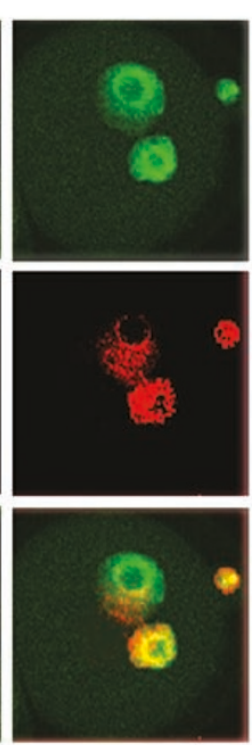

PN4/5
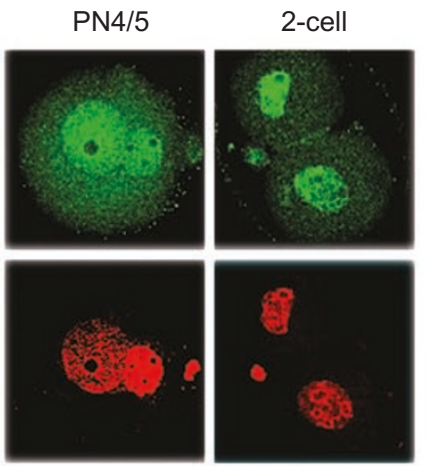

9.
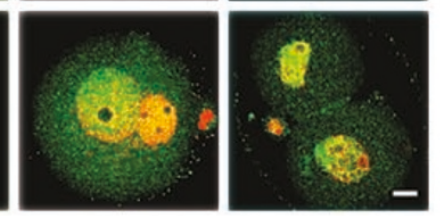

$\mathrm{PN} 4 / 5$

2-cell

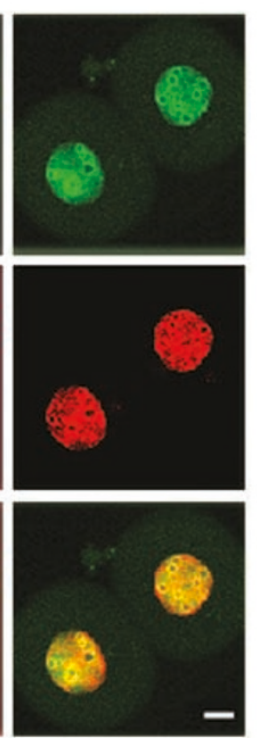

$-$

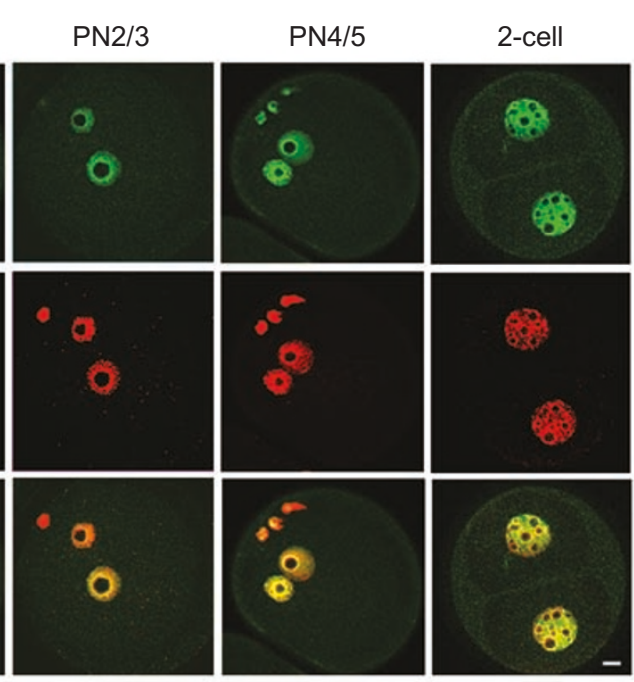



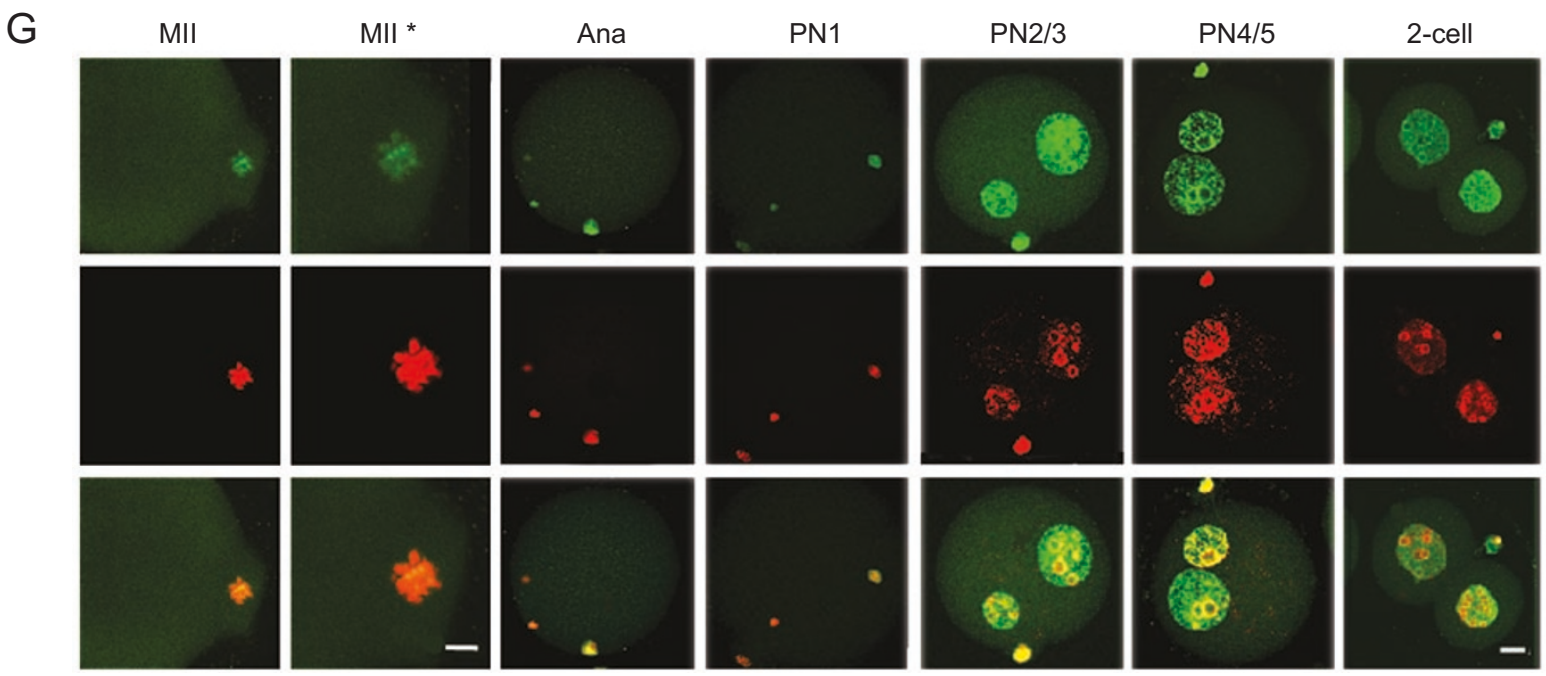

Figure 6 Re-association between CFs and chromatin/pronuclei after fertilization. TFIIB (A) and AP2 $\alpha$ (B), BRG1 (C), SRG3 (D), HDAC2 (E) became detected on chromatin during pronuclear formation. TFIIB was also detected on structures in the vicinity of chromosomes and AP $2 \alpha$, on the sperm chromatin, before meiosis was completed. Signals of the five CFs were co-localized with chromatin in nuclei of all stages, indicating that these CFs became re-associated with chromatin after entering the nuclei. HP1 $\beta$ (F) and $\mathrm{AcH} 4$ (G) were detected on the meiotic chromosomes and pronuclei of all stages examined. Bar=10 and $5 \mu \mathrm{m}$ in low or high (*) magnitude, respectively. See Supplementary information III for more details with these CFs.

\section{Many CFs critical for transcription remained undetectable on chromosomes before pronuclear formation}

Antibody staining of fertilized one-cell embryos revealed that most CFs considered, including TBP, TAF1, TAF4, TFIIA, TFIIB, Pol II, BRF1, AP2 $\alpha$, BRG1, SRG3, INI1, YY1, HDAC1, HDAC2, MeCP2, MBD2, remained undetectable on chromosomes before the completion of meiosis in one-cell embryos (Figure 6, Supplementary information III, and Table 4). These results, together with the fact that transcription remains silent at the time, suggested that the transcription apparatus and other multiprotein regulatory complexes had not been assembled onto chromatin yet. Meanwhile, HP1 $\alpha$, HP1 $\beta$, TOPOII $\alpha$, TOPOII $\beta$, and $\mathrm{AcH} 4$ were observed to be associated with chromosomes, on either a portion or the entire body of them.

Several CFs were detected to be associated with structures located in the vicinity of condensed chromosomes in later meiotic phases. These included TBP, TAF1, TFIIB, YY1, MBD2, and TOPOII $\beta$ (Figure 6 and Supplementary information III). The labeling of these subcellular structures in one-cell embryos was specific and could be blocked respectively by CF-specific peptides (Supplementary information III-23 and data not shown). The identities of these structures were not analyzed, but their location and morphology suggested that they could be either remnant of nuclei or components of the spindle. Being bound to structures in the vicinity of chromosomes might facilitate the entry of those CFs into pronuclei.

\section{Reloading of CFs onto chromatin before the first cell divi- sion}

Approximately 4 to $5 \mathrm{~h}$ after fertilization, pronuclei begin to emerge in a proportion of the embryos. HP $1 \alpha$, HP $1 \beta$, TOPOII $\alpha$, TOPOII $\beta$, and $\mathrm{AcH} 4$, which were on chromatin before pronuclear formation, continued to be positive in pronuclei of all stages. The other $16 \mathrm{CFs}$ examined (TBP, TAF1, TAF4, TFIIA, TFIIB, Pol II, BRF1, AP2 $\alpha$, BRG1, SRG3, INI1, YY1, HDAC1, HDAC2, MECP2, and MBD2) became detectable in nuclei shortly after pronuclear formation, in the PN1, PN2, or PN3 stage. All 21 CFs were present thereafter in the nuclei in PN4, PN5, 2-cell (Figure 6, Supplementary information III, and Table 4) [23, 43, 56, 57], and other pre-implantation stages (data not shown). Quantitative assays for TBP [23], TRF3 [43], TAF1 [56], SRG3 [57] in previous experiments indicated that nuclear concentrations of these CFs increased steadily and reached significantly high levels before ZGA.

Thus, our immunochemical analyses suggested that the cytoplasm of early one-cell embryos did not favor the association between chromatin and transcription factors or regulators. To further confirm this, NIH3T3 cells expressing GFP-TBP or GFP-HP1 $\beta$ were transplanted to intact MII eggs. As expected, GFP-HP1 $\beta$ migrated out of NIH3T3 nucleus and were bound to maternal chromo- 
Table 4 A summary in subcellular distribution of CFs in fertilized one-cell embryos

\begin{tabular}{|c|c|c|c|c|c|c|}
\hline & MII & Anaphase & PN1 & PN2/PN3 & PN4/PN5 & 2-cell \\
\hline$\overline{\mathrm{TBP}^{2}}$ & $-{ }^{1} 41^{5}$ & -35 & -21 & $+57 /-20$ & +38 & +21 \\
\hline $\mathrm{TAF} 1^{2}$ & -40 & -35 & -44 & $+44 /-49$ & +17 & +13 \\
\hline TAF4 & -31 & -29 & -22 & $+25 /-12$ & +12 & +14 \\
\hline TFIIA & -48 & -15 & -25 & $+22 /-17$ & +27 & +16 \\
\hline TFIIB $^{2,3}$ & -57 & -41 & -24 & $+39 /-13$ & +19 & +11 \\
\hline ol II & -59 & -34 & -19 & $+14 /-39$ & +11 & +24 \\
\hline BRF1 & -44 & -35 & -25 & $+23 /-42$ & +11 & +35 \\
\hline $\mathrm{AP} 2 \alpha^{3}$ & -22 & -16 & $+1 /-18$ & $+29 /-36$ & +12 & +14 \\
\hline BRG1 & -50 & -34 & -23 & $+37 /-7$ & +24 & +16 \\
\hline $\mathrm{SRG}^{3}$ & -72 & -34 & $+8 /-14$ & +42 & +23 & +10 \\
\hline $\mathrm{INI}^{3}$ & -88 & -43 & $+2 /-17$ & $+32 /-7$ & +18 & +10 \\
\hline$Y Y 1^{2}$ & -20 & -25 & -19 & $+31 /-7$ & +24 & +20 \\
\hline HDAC1 & -70 & -48 & -19 & $+32 /-14$ & +32 & +11 \\
\hline $\mathrm{HDAC}^{2,3}$ & -12 & -20 & $+6 /-23$ & +66 & +24 & +10 \\
\hline $\mathrm{MECP}^{3,4}$ & -41 & -20 & $+6 /-23$ & +39 & +8 & +13 \\
\hline MBD2a, $b^{2}$ & -32 & -10 & -12 & $+41 /-1$ & +8 & +10 \\
\hline $\mathrm{HP} 1 \alpha^{3}$ & +44 & +22 & +11 & +37 & +19 & +12 \\
\hline$H P 1 \beta^{3}$ & +22 & +19 & +33 & +45 & +18 & +12 \\
\hline TOPII $\alpha^{3}$ & +28 & $+11 /-10$ & $+6 /-13$ & $+61 /-9$ & +12 & +14 \\
\hline TOPII $\beta^{2,3}$ & +33 & $+10 /-2$ & +40 & +74 & +30 & +11 \\
\hline $\mathrm{Ac}-\mathrm{H} 4^{3}$ & $+25 /-4$ & +22 & +11 & +39 & +18 & +33 \\
\hline
\end{tabular}

${ }^{1}+$ and - : signal was or was not detected on chromosomes or PNs, respectively.

${ }^{2}$ Signal is also on structures in the vicinity of chromosomes.

${ }^{3}$ Signal is also on the sperm chromatin in anaphase.

${ }^{4}$ Signal is in the centromeric region of chromosomes in MII eggs.

${ }^{5}$ The numbers of embryos with the phenotype (all embryos examined are included in the table, i.e., when only one number is in the column, $100 \%$ of the embryos had the particular phenotype).

somes in anaphase, telophase, and PNs of all stages while GFP-TBP remained unbound to maternal chromatin until pronuclei were clearly formed (Figure 7). Results from the fusion protein study confirmed that the cytoplasm of early one-cell embryos does not promote chromatin binding of the transcription factor TBP and it became re-associated with chromatin after pronuclear formation. It was noticed that both maternal and somatic chromatin transformed into PNs, and GFP-TBP and GFP-HP1 $\beta$ entered all of them indiscriminately (Figure 7). The result indicated that binding of CFs to chromatin in one-cell embryos was not influenced by the individual developmental background of the chromatin.

\section{Discussion}

In this study, we show that a broad range of CFs essential for transcription activation and regulation was dissociated from chromatin during the first meiotic cycle, remained physically separated from chromatin for a prolonged time, and became re-associated with chromatin after pronuclear formation.

In agreement with the previous study [29], we found that a substantial amount of TAF1, BRG1, and SRG3 remained bound to chromatin in the $\mathrm{SN}$ stage in addition to members of the Pol I transcription complex. It is also entirely possible that even those CFs judged as "dissociated" in this assay may have residual amounts left on condensed chromatin. However, it has been well documented that the wholeness (intactness) of the transcription machinery and regulatory mechanism is essential for an active transcription program. The absence of even a single essential transcription or regulatory factor can inactivate transcription. For example, deletion of TBP eliminates completely transcription mediated by both Pol I and Pol III [73], and deletion of maternal BRG1 diminishes ZGA [10]. Therefore, dissociation of most molecules of TBP, TRF3, TAF4, TFIIB, BRF1, Pol II, AP2 $\alpha$, INI1, etc. from chromatin is likely sufficient to prevent the formation of an intact transcription apparatus. It is likely that the list of CFs being displaced during meiosis extends far beyond those examined so far. In addition, changes in chemical modifications and functional status of critical transcription factors [5, 24-27] could prompt disassembly of the transcription apparatus. When contacts between chromatin and a broad range of critical transcription factors and regulatory factors are disrupted, transcription cannot occur. Based on these observations, we propose that a genome-wide CF dissociation that leads to disassembly of the transcription machinery and regulatory mechanism is the primary cause of transcription inactivation in oocytes.

Approximately by the end of the first meiotic cycle, a majority of critical transcription factors and regulators are dissociated from the chromatin. The maternal transcription program no longer exists. Most CFs remain undetectable on chromatin after fertilization by the current assay and become detectable in pronuclei around the PN3 stage, at a time when the formation of the pronuclear envelopes is completed [58]. In previous studies, kinetochore proteins [59], SP1, TBP [23], TAF1 [56], UBF, RPA53/PAF53, RPA116 [29], Ring1B, Ph1 [28], and SRG3 [57] are found to enter pronuclei at a similar time. Re-association between chromatin and CFs is followed temporally by DNA replication $[34,35]$, and at the end of replication, ZGA takes place [34]. Quantitative analyses of SP1, TBP [23], TAF1 [56], and SRG3 [57] in one-cell embryos have revealed that nuclear concentrations of these CFs increase steadily during pronuclear formation and migration, and reach significant 
A
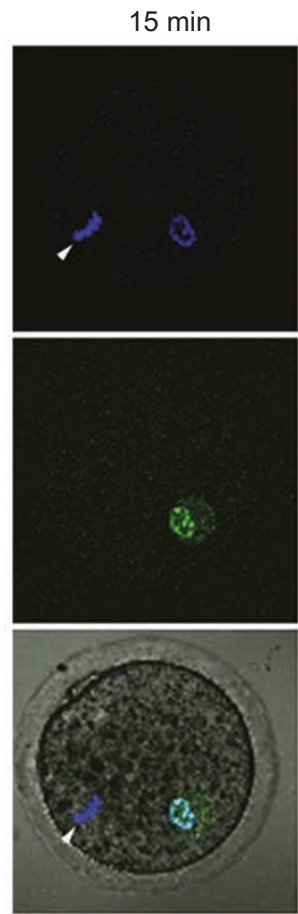

B

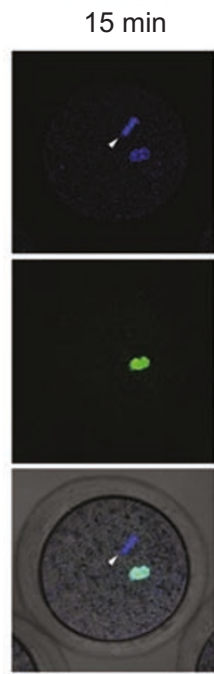

$2 \mathrm{~h}$
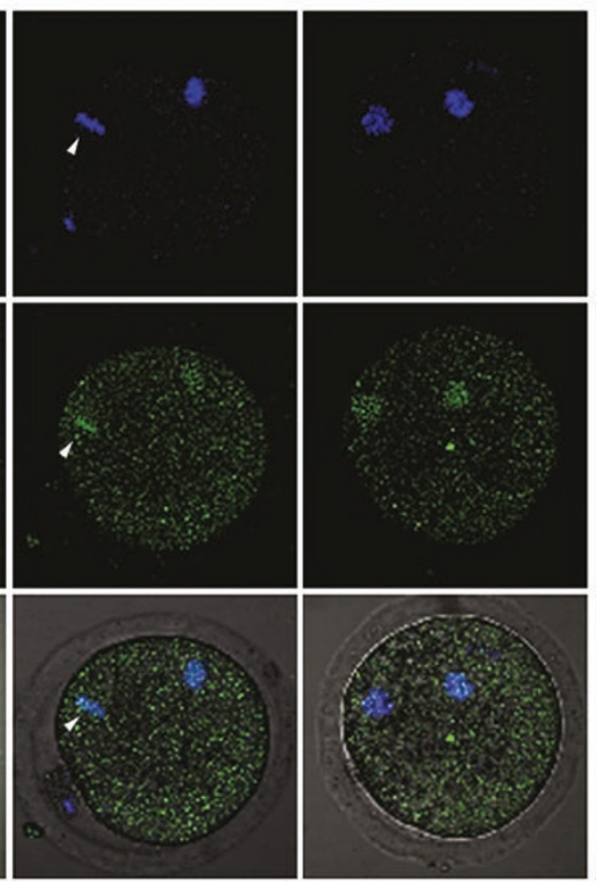
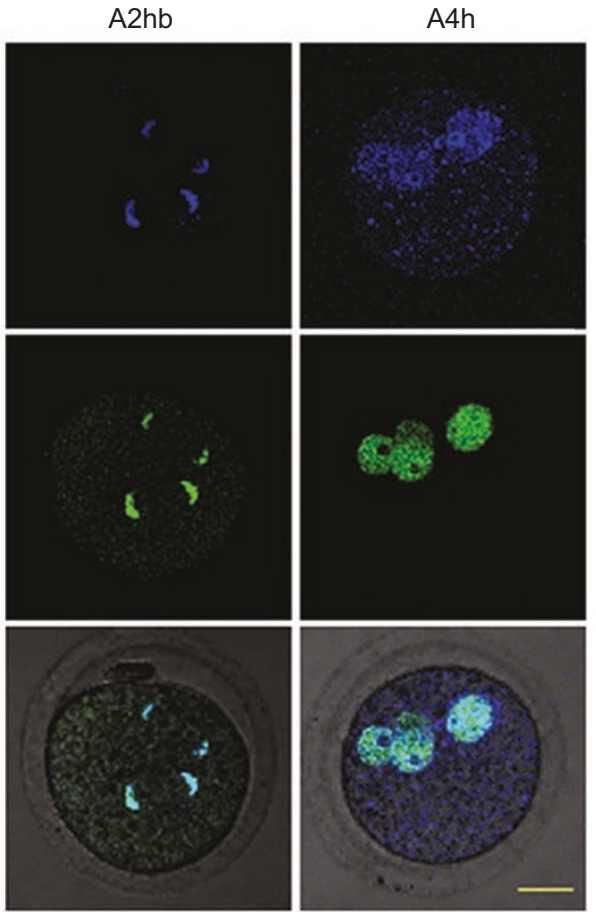

2ha

$2 \mathrm{hb}$

A2ha
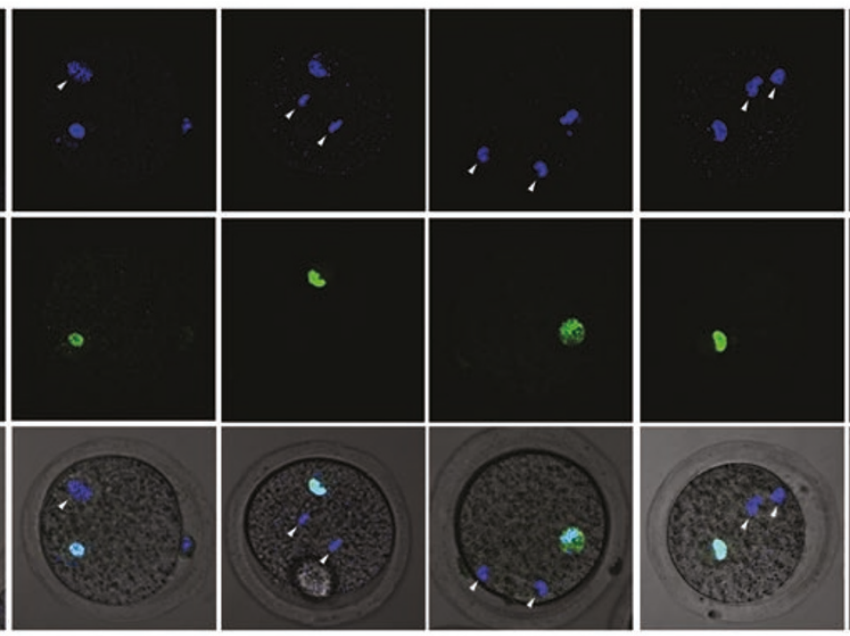

A4ha

A4hb

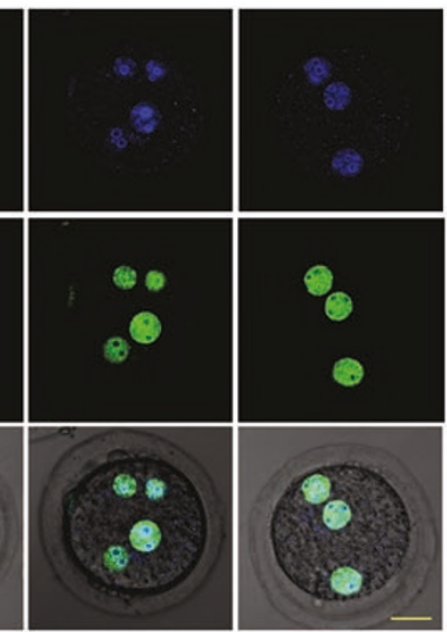

Figure 7 Subcellular distribution of GFP-HP1 $\beta$ and GFP-TBP in intact MII oocytes. An NIH3T3 expressing either GFP-HP1 $\beta$ (A) or GFP-TBP (B) (green) was transplanted into intact MII eggs. GFP-HP1 $\beta$ was not seen on maternal chromosomes (arrow heads) shortly after $\mathrm{nt}(15 \mathrm{~min})$ and became associated with chromosomes at $2 \mathrm{~h}$ after $\mathrm{nt}(2 \mathrm{~h})$ and remained associated thereafter (A2 $\mathrm{h}$ and A4 h). In the parallel experiment, GFP-TBP was not associated with maternal chromatin until pronuclear formation. Note that both the maternal chromatin and somatic nuclei formed pronuclei in some embryos and GFP-HP1 $\beta$ and GFP-TBP entered them non-discriminately. Blue, Hoechest 33342 staining (top row). $15 \mathrm{~min}$ and $2 \mathrm{~h}$ indicate times after transplantation. A2 h, A4 h indicate hours after chemical activation of reconstructed embryos. Bar $=10 \mu \mathrm{m}$.

high levels by ZGA. The fact that ZGA does not take place immediately after re-association between chromatin and $\mathrm{CFs}$, but approximately 4 to $5 \mathrm{~h}$ later, may be explained by the possibilities that on-going DNA replication may prevent transcription, and that transcription is not able to take place before all essential CFs have reached threshold levels and a functional basal transcription machine and regulatory mechanism are assembled. Based on these observations and analyses, we conclude that transcription status of the chromatin correlates in general with dynamic 
changes in its relationship with CFs. Transcription ceases when chromatin loses contact with a vast number of CFs and resumes after pronuclei/chromatin regains most $\mathrm{CFs}$ over threshold levels. Transcription silence in oocytes and early embryos, for most part, reflects the inert status of the chromatin after the maternal transcription program is removed and before the zygotic equivalent is re-installed. The period of transcription silence is further extended by the first DNA replication.

Transcription silence is observed at the transition point of two generations across diverse species [60], and even the timings for transcription cessation and reactivation are also conserved. For example, transcription ceases during the first meiotic cycle in both Caenorhabditis elegans [61, 62] and mouse [1-5]. Major ZGA occurs after fertilization in blastomeres, after a few (most mammals) or up to 12 divisions (Xenopus and flies). If this transcription silence is for most part a result of the inert status of the chromatin after disassembly of the parental transcription program and before the assembly of the zygotic equivalent as proposed above, it implies that the "erase-and-rebuild" process is an evolutionary conserved mechanism widely used for building up new life cycles.

Transcription also ceases during mitosis and most transcription factors are dissociated from mitotic chromosomes [63-66]. While transcription silence during mitosis and meiosis shares many similarities, they differ in at least one fundamental aspect. The gene expression pattern and phenotype of the cell are generally maintained after mitosis, but they are changed after meiosis. Two mechanisms may potentially lead to the switch in transcription profiles during meiosis. First, erasure of the maternal transcriptional program during meiosis may be more thorough than that during mitosis, which could be achieved through varying the type and amount of CFs being displaced, and through additional reprogramming processes, such as global demethylation [67-69] and deacetylation [70], which occur during oogenesis. The combinatorial effect of all these processes can lead to functional erasure of the maternal epigenetic program. Indeed, although a haploid set of maternal chromatin is passed on to the zygote, it never encodes for maternal-specific traits after meiosis. Second, mature oocytes contain most (if not all) components needed for assembling a zygotic transcription program [71], while the cytoplasm of somatic cells may contain components for re-assembling the transcription program specific for a certain differentiation stage.

Oogenesis is a special period when several processes take place aiming to eliminate epigenetic information installed in the maternal chromatin. In parallel to CF dissociation that clear memories carried on by peptide factors, genome-wide demethylation [67-69] and a global histone deacetylation [70] remove memories maintained through covalent modifications. We suggest that the convergent effect of these, and most likely other, reprogramming processes serves to erase the maternal-specific epigenetic program, so as to create a relatively naïve chromatin that can be critical for building the program for the next life cycle. We propose that this is an important function of oogenesis, which has not been fully appreciated previously. It is also worth noting that binding of multiprotein complexes to chromatin is fundamental not only to transcription but also to all known nuclear processes, e.g. DNA replication, recombination, and repair. It is likely that the "erase-andrebuild" process reprograms not only the transcription profile but also other nuclear processes. The demonstration that the frequency of DNA replication origins of a somatic nucleus is reset to that of embryonic cells by egg extract [53] provides evidence supporting the speculation.

In summary, we propose that the maternal chromatin is reprogrammed to the zygotic chromatin using an acrossthe-board "erase-and-rebuild" process that takes place in two phases. First, a genome-wide CF displacement disrupts contacts between chromatin and CFs and erases the existing transcription program and other epigenetic information from the maternal chromatin. This, in combination with other reprogramming processes [67-70], will transform the maternal chromatin into a relatively naïve status, providing a base for building a new life cycle. Second, most CFs are loaded to chromatin shortly after pronuclear formation. This marks the beginning of an active period to assemble a zygotic developmental program. The mature oocyte contains most, if not all, necessary factors for the assembling process.

\section{Acknowledgement}

We are grateful to Drs Yun-Bo Shi, Yingzi Yang, and Paul Zhou for criticizing the manuscript, Dr Shangang Li for assistance in nuclear transfer procedure, Wei Su for art work, Youming Zhu for cell culture, and Xun Gong, Hui Ding, and Wei Liu for assistance in immunochemistry, Fengying Li and Wanli Li for animal care, and Ayong Yan for nuclear transfer into MII oocytes. We also apologize for citing reviews instead of original publications in some places due to space limitations. Please look in the reviews for original papers.

The study was supported by grants from National Basic Research Program of China (973 Program) (No. 001CB509903, 001CB509904), Hi-Tech Research and Development Program of China (863 Program) (No. 2001AA216121, 2004AA205010), Science and Technology Committee of Shanghai Municipality (No. 99DJ14002, 00DJ1 4033, 01DJ14003, 03DJ14017), Shanghai Municipal Education Commission (No. T0205) and Shanghai Jiao Tong University, School of Medicine. 
Contributions: Ruizhen $\mathrm{Li}$ is responsible for most analyses in GV oocytes, Feng Sun and Junke Zheng for analyses in one-cell embryos, Tianlong Gao and Haiyan Fang for GFP-CF analyses, Xuejin Chen and Wenqin Ying for nuclear transfer procedures. Feng Sun assisted in manuscript writing. Hui Z Sheng is responsible for development of the model, project planning, and most manuscript writing.

\section{References}

1 Bouniol-Baly C, Hamraoui L, Guibert J, Beaujean N, Szollosi MS, Debey P. Differential transcriptional activity associated with chromatin configuration in fully grown mouse germinal vesicle oocytes. Biol Reprod 1999; 60:580-587.

2 De La Fuente R, Viveiros MM, Burns KH, Adashi EY, Matzuk MM, Eppig JJ. Major chromatin remodeling in the germinal vesicle $(\mathrm{GV})$ of mammalian oocytes is dispensable for global transcriptional silencing but required for centromeric heterochromatin function. Dev Biol 2004; 275:447-458..

3 De La Fuente R. Chromatin modifications in the germinal vesicle (GV) of mammalian oocytes. Dev Biol 2006; 292:1-12.

4 De La Fuente R, Eppig JJ. Transcriptional activity of the mouse oocyte genome: companion granulosa cells modulate transcription and chromatin remodeling. Dev Biol 2001; 229:224-236.

5 Miyara F, Migne C, Dumont-Hassan M, et al. Chromatin configuration and transcriptional control in human and mouse oocytes. Mol Reprod Dev 2003; 64:458-470.

6 Ram PT, Schultz RM. Reporter gene expression in G2 of the 1-cell mouse embryo. Dev Biol 1993; 156:552-556.

7 Aoki F, Worrad DM, Schultz RM. Regulation of transcriptional activity during the first and second cell cycles in the preimplantation mouse embryo. Dev Biol 1997; 181:296-307.

8 Schultz RM. The molecular foundations of the maternal to zygotic transition in the preimplantation embryo. Hum Reprod Update 2002; 8:323-331.

9 Bouniol C, Nguyen E, Debey P. Endogenous transcription occurs at the 1-cell stage in the mouse embryo. Exp Cell Res 1995; 218:57-62.

10 Bultman SJ, Gebuhr TC, Pan H, Svoboda P, Schultz RM, Magnuson T. Maternal BRG1 regulates zygotic genome activation in the mouse. Genes Dev 2006; 20:1744-1754.

11 Surani MA. Nuclear reprogramming by human embryonic stem cells. Cell 2005; 122:653-654.

12 Hochedlinger K, Jaenisch R. Nuclear reprogramming and pluripotency. Nature 2006; 441:1061-1067.

13 Gurdon JB, Byrne JA. The first half-century of nuclear transplantation. Biosci Rep 2004; 24:545-557.

14 Edgar BA, Schubiger G. Parameters controlling transcriptional activation during early Drosophila development. Cell 1986; 44:871-877.

15 Kimelman D, Kirschner M, Scherson T. The events of the midblastula transition in Xenopus are regulated by changes in the cell cycle. Cell 1987; 48:399-407.

16 Yasuda GK, Schubiger G. Temporal regulation in the early embryo: is MBT too good to be true? Trends Genet 1992; 8:124127.

17 Newport J, Kirschner M. A major developmental transition in early Xenopus embryos: II. Control of the onset of transcription.
Cell 1982; 30:687-696.

18 Prioleau MN, Huet J, Sentenac A, Mechali M. Competition between chromatin and transcription complex assembly regulates gene expression during early development. Cell 1994; 77:439449.

19 Prioleau MN, Buckle RS, Mechali M. Programming of a repressed but committed chromatin structure during early development. EMBO J 1995; 14:5073-5084.

20 Almouzni G, Wolffe AP. Constraints on transcriptional activator function contribute to transcriptional quiescence during early Xenopus embryogenesis. EMBO J 1995; 14:1752-1765.

21 Bell P, Scheer U. Developmental changes in RNA polymerase I and TATA box-binding protein during early Xenopus embryogenesis. Exp Cell Res 1999; 248:122-135.

22 Veenstra GJ, Destree OH, Wolffe AP. Translation of maternal TATA-binding protein mRNA potentiates basal but not activated transcription in Xenopus embryos at the midblastula transition. Mol Cell Biol 1999; 19:7972-7982.

23 Worrad DM, Ram PT, Schultz RM. Regulation of gene expression in the mouse oocyte and early preimplantation embryo: developmental changes in Sp1 and TATA box-binding protein, TBP. Development 1994; 120:2347-2357.

24 Parfenov VN, Pochukalina GN, Davis DS, Reinbold R, Scholer HR, Murti KG. Nuclear distribution of Oct-4 transcription factor in transcriptionally active and inactive mouse oocytes and its relation to RNA polymerase II and splicing factors. J Cell Biochem 2003; 89:720-732.

25 Parfenov VN, Davis DS, Pochukalina GN, Kostyuchek D, Murti KG. Nuclear distribution of RNA polymerase II in human oocytes from antral follicles: dynamics relative to the transcriptional state and association with splicing factors. J Cell Biochem 2000; 77:654-665.

26 Bellier S, Chastant S, Adenot P, Vincent M, Renard JP, Bensaude O. Nuclear translocation and carboxyl-terminal domain phosphorylation of RNA polymerase II delineate the two phases of zygotic gene activation in mammalian embryos. EMBO J 1997; 16:6250-6262.

27 Gebara MM, Sayre MH, Corden JL. Phosphorylation of the carboxy-terminal repeat domain in RNA polymerase II by cyclin-dependent kinases is sufficient to inhibit transcription. J Cell Biochem 1997; 64:390-402.

28 Miyagishima H, Isono K, Fujimura Y, et al. Dissociation of mammalian Polycomb-group proteins, Ring1B and Rae28/Ph1, from the chromatin correlates with configuration changes of the chromatin in mitotic and meiotic prophase. Histochem Cell Biol 2003; 120:111-119.

29 Zatsepina OV, Bouniol-Baly C, Amirand C, Debey P. Functional and molecular reorganization of the nucleolar apparatus in maturing mouse oocytes. Dev Biol 2000; 223:354-370.

30 Kornberg RD. Mediator and the mechanism of transcriptional activation. Trends Biochem Sci 2005; 30:235-239.

31 Lemon B, Tjian R. Orchestrated response: a symphony of transcription factors for gene control. Genes Dev 2000; 14:25512569.

32 Orphanides G, Reinberg D. A unified theory of gene expression. Cell 2002; 108:439-451.

33 Green MR. Eukaryotic transcription activation: right on target. Mol Cell 2005; 18:399-402.

34 Bouniol-Baly C, Nguyen E, Besombes D, Debey P. Dynamic 
organization of DNA replication in one-cell mouse embryos: relationship to transcriptional activation. Exp Cell Res 1997; 236:201-211.

35 Adenot PG, Mercier Y, Renard JP, Thompson EM. Differential $\mathrm{H} 4$ acetylation of paternal and maternal chromatin precedes DNA replication and differential transcriptional activity in pronuclei of 1-cell mouse embryos. Development 1997; 124:4615-4625.

36 Chatot CL, Ziomek CA, Bavister BD, Lewis JL, Torres I. An improved culture medium supports development of random-bred 1-cell mouse embryos in vitro. J Reprod Fertil 1989; 86:679688.

37 Wakayama T, Perry AC, Zuccotti M, Johnson KR, Yanagimachi R. Full-term development of mice from enucleated oocytes injected with cumulus cell nuclei. Nature 1998; 394:369-374.

38 Debey P, Szollosi MS, Szollosi D, Vautier D, Girousse A, Besombes D. Competent mouse oocytes isolated from antral follicles exhibit different chromatin organization and follow different maturation dynamics. Mol Reprod Dev 1993; 36:59-74.

39 Mattson BA, Albertini DF. Oogenesis: chromatin and microtubule dynamics during meiotic prophase. Mol Reprod Dev 1990; 25:374-83.

40 Wickramasinghe D, Ebert KM, Albertini DF. Meiotic competence acquisition is associated with the appearance of M-phase characteristics in growing mouse oocytes. Dev Biol 1991; 143:162172.

41 Zuccotti M, Piccinelli A, Giorgi Rossi P, Garagna S, Redi CA. Chromatin organization during mouse oocyte growth. Mol Reprod Dev 1995; 41:479-485.

42 Kim JM, Ogura A, Nagata M, Aoki F. Analysis of the mechanism for chromatin remodeling in embryos reconstructed by somatic nuclear transfer. Biol Reprod 2002; 67:760-766.

43 Yang Y, Cao J, Huang L, Fang HY, Sheng HZ. Regulated expression of TATA-binding protein-related factor 3 (TRF3) during early embryogenesis. Cell Res 2006; 16:610-621.

44 Kadonaga JT. Regulation of RNA polymerase II transcription by sequence-specific DNA binding factors. Cell 2004; 116:247257.

45 Soutoglou E, Talianidis I. Coordination of PIC assembly and chromatin remodeling during differentiation-induced gene activation. Science 2002; 295:1901-1904.

46 Fry CJ, Peterson CL. Transcription. Unlocking the gates to gene expression. Science 2002; 295:1847-1848.

47 Thomas MJ, Seto E. Unlocking the mechanisms of transcription factor YY1: are chromatin modifying enzymes the key? Gene 1999; 236:197-208.

48 Varga-Weisz PD, Becker PB. Chromatin-remodeling factors: machines that regulate? Curr Opin Cell Biol 1998; 10:346-353.

49 Tate P, Skarnes W, Bird A. The methyl-CpG binding protein $\mathrm{MeCP} 2$ is essential for embryonic development in the mouse. Nat Genet 1996; 12:205-208.

50 Bakshi RP, Galande S, Muniyappa K. Functional and regulatory characteristics of eukaryotic type II DNA topoisomerase. Crit Rev Biochem Mol Biol 2001; 36:1-37.

51 Maison C, Almouzni G. HP1 and the dynamics of heterochromatin maintenance. Nat Rev Mol Cell Biol 2004; 5:296-304.

52 Mondal N, Parvin JD. DNA topoisomerase IIalpha is required for RNA polymerase II transcription on chromatin templates. Nature 2001; 413:435-438.

53 Lemaitre JM, Danis E, Pasero P, Vassetzky Y, Mechali M. Mitotic remodeling of the replicon and chromosome structure. Cell 2005 123:787-801.

54 Chen D, Hinkley CS, Henry RW, Huang S. TBP dynamics in living human cells: constitutive association of TBP with mitotic chromosomes. Mol Biol Cell 2002; 13:276-284.

55 Chen D, Dundr M, Wang C, et al. Condensed mitotic chromatin is accessible to transcription factors and chromatin structural proteins. J Cell Biol 2005; 168:41-54.

56 Wang K, Sun F, Sheng HZ. Regulated expression of TAF1 in 1-cell mouse embryos. Zygote 2006; 14:209-215.

57 Sun F, Tang F, Yan AY, Fang HY, Sheng HZ. Expression of SRG3, a chromatin remodeling factor, in the mouse oocyte and early preimplantation embryos. Zygote 2007; 15:1-10.

58 Luthardt FW, Donahue RP. Pronuclear DNA synthesis in mouse eggs. An autoradiographic study. Exp Cell Res 1973; 82:143151.

59 Schatten G, Simerly C, Palmer DK, et al. Kinetochore appearance during meiosis, fertilization and mitosis in mouse oocytes and zygotes. Chromosoma 1988; 96:341-352.

60 Davidson EH. Gene Activity in Early Development. Orlando, FL: Academic Press, 1986.

61 Kelly WG, Schaner CE, Dernburg AF, et al. X-chromosome silencing in the germline of C. elegans. Development 2002; 129:479-492.

62 Schisa JA, Pitt JN, Priess JR. Analysis of RNA associated with P granules in germ cells of C. elegans adults. Development 2001; 128:1287-1298.

63 Taylor JH. Nucleic acid synthesis in relation to the cell division cycle. Ann NY Acad Sci 1960; 90:409-421.

64 Prescott DM, Bender MA. Synthesis of RNA and protein during mitosis in mammalian tissue culture cells. Exp Cell Res 1962; 26:260-268.

65 Littau VC, Allfrey VG, Frenster JH, Mirsky AE. Active and inactive regions of nuclear chromatin as revealed by electron microscope autoradiography. Proc Natl Acad Sci USA 1964; 52:93-100.

66 Johnson TC, Holland JJ. Ribonucleic acid and protein synthesis in mitotic HeLa cells. J Cell Biol 1965; 27:565-574.

67 Reik W, Dean W, Walter J. Epigenetic reprogramming in mammalian development. Science 2001; 293:1089-1093.

68 Santos F, Zakhartchenko V, Stojkovic M, et al. Epigenetic marking correlates with developmental potential in cloned bovine preimplantation embryos. Curr Biol 2003; 13:1116-1121.

69 Surani MA. Reprogramming of genome function through epigenetic inheritance. Nature 2001; 414:122-128.

70 Kim JM, Liu H, Tazaki M, Nagata M, Aoki F. Changes in histone acetylation during mouse oocyte meiosis. J Cell Biol 2003; 162:37-46.

71 Gao T, Zheng J, Xing F, et al. Nuclear reprogramming: the strategy used in normal development is also used in somatic cell nuclear transfer and parthenogenesis. Cell Res 2007; 17:135150.

72 Imhof A, Wolffe AP. Transcription: gene control by targeted histone acetylation. Curr Biol 1998; 8:R422-R424.

73 Martianov I, Viville S, Davidson I. RNA polymerase II transcription in murine cells lacking the TATA binding protein. Science 2002; 298:1036-1039.

(Supplementary Information is linked to the online version of the paper on the Cell Research website.) 